\title{
Postures et impostures énonciatives. Notes sur le discours polyphonique
}

\section{Federico Bravo}

\section{Resumen}

En el presente estudio se ofrece una descripción de los mecanismos enunciativos mediante los cuales el hablante, distanciándose de su propia producción verbal, se substrae a la triple demarcación yo-aquí-ahora en que se funda el acto lingüístico, para oponerle un sistema de referenciación autónomo que, construido a partir de un doble rechazo - rechazo por parte del enunciador a ocupar el presente espacio-temporal del locutar e, inversamente, rechazo por parte del locutor a asumir el punto de vista del enunciador -, instituye en el discurso un orden enunciativo coherente y rigurosamente estructurado.

\section{Résumé}

La présente étude offre une description des mécanismes énonciatifs par lesquels le sujet parlant, en se distançant de sa propre production discursive, échappe au triple repère je-ici-maintenant fondateur de l'acte de parole, pour lui opposer un système de référenciation autonome qui, édifié sur un double refus - refus de l'énonciateur à partager le présent spatiotemporel du locuteur et, inversement, refus du locuteur à adopter le point de vue de l'énonciateur -, instaure dans le discours un ordre énonciatif cohérent et rigoureusement structuré.

\section{Citer ce document / Cite this document :}

Bravo Federico. Postures et impostures énonciatives. Notes sur le discours polyphonique. In: Bulletin Hispanique, tome 95, n¹, 1993. pp. 59-97;

doi : https://doi.org/10.3406/hispa.1993.4785

https://www.persee.fr/doc/hispa_0007-4640_1993_num_95_1_4785

Fichier pdf généré le 26/05/2018 


\title{
POSTURES ET IMPOSTURES ÉNONCIATIVES Notes sur le discours polyphonique
}

\author{
Federico BRAVO
}

Où maintenant? Quand maintenant? Qui maintenant? Samuel BECKETT, L'Innommable.

La présente étude offre une description des mécanismes énonciatifs par lesquels le sujet parlant, en se distançant de sa propre production discursive, échappe au triple repère jeici-maintenant fondateur de l'acte de parole, pour lui opposer un système de référenciation autonome qui, édifié sur un double refus - refus de l'énonciateur à partager le présent spatio-temporel du locuteur et, inversement, refus du locuteur à adopter le point de vue de l'énonciateur -, instaure dans le discours un ordre énonciatif cohérent et rigoureusement structuré.

En el presente estudio se ofrece una descripción de los mecanismos enunciativos mediante los cuales el hablante, distanciándose de su propia producción verbal, se substrae a la triple demarcación yo-aquí-ahora en que se funda el acto lingüístico, para oponerle un sistema de referenciación autónomo que, construido a partir de un doble rechazo - rechazo por parte del enunciador a ocupar el presente espacio-temporal del locutar e, inversamente, rechazo por parte del locutor a asumir el punto de vista del enunciador -, instituye en el discurso un orden enunciativo coherente y rigurosamente estructurado.

Tout acte de parole suppose un énonciateur, un lieu de parole et un moment d'énonciation. A ce triple repère l'énonciateur n'est pas libre de se soustraire : lorsqu'il parle, il le fait nécessairement en un lieu et dans un temps qui déterminent son présent de locution. Si telle est invariablement la situation qui accompagne toute énonciation, il est des situations de parole dans lesquelles le locuteur semble s'affranchir tantôt de son présent spatio-temporel, tantôt de sa position locutive en tant que producteur de l'assertion. Les remarques qui suivent, largement inspirées des travaux qui, au cours des dernières décennies, ont contribué à l'élaboration de la notion de polyphonie, ont pour objet d'analyser quelques-unes des stratégies qui permettent de contourner

B. Hi., T. 95, 1993, n० 1, p. 59 à 97. 
cet incontournable de l'énonciation qu'est le triple repère je-icimaintenant. Notre propos n'est pas d'apporter un éclairage nouveau à une théorie dont l'intérêt et la pertinence ne sont plus à démontrer, mais de réexaminer, a la lumière de ses principaux acquis, un certain nombre de questions axées autour de deux problèmes majeurs de l'énonciation polyphonique, à savoir : le dédoublement spatiotemporel de l'instance de locution et l'énonciation ironique.

\section{I. - « JE » EST AILLEURS}

La combinatoire syntagmatique des morphèmes déictiques dans l'énoncé est, comme la plupart des grammaires s'attachent à l'expliquer, soumise à des règles d'intégration syntaxique dont la transgression a pour effet, selon ces mêmes grammaires, de porter atteinte a la cohésion interne de la phrase. L'observation directe des réalisations discursives fait souvent apparaître cependant des distorsions notables entre la combinatoire «idéale " que postulent les grammaires et celle, apparemment disparate, dont témoignent certains emplois, tant à l'écrit qu'à l'oral. Lorsqu'ils ne sont pas simplement considérés comme des "ratages" de la parole, ces emplois plus ou moins "irréguliers " sont soit mis sur le compte de l'« expressivité " soit imputés à des défaillances survenues au cours de l'énonciation. Aussi expédie-t-on souvent le problème en invoquant tantôt la "marginalité " du phénomène - comme si le nombre des témoignages était à lui seul un critère suffisant pour exclure du champ de la description des faits de langue pourtant attestés -, tantôt sa visée "stylistique "-comme si les effets de sens générés par les mécanismes linguistiques mobilisés étaient dissociables des structures mentales qui les sous-tendent -.

Nous avons choisi, quant a nous, de voir dans certaines de ces réalisations l'effet d'un dédoublement polyphonique qui, scindant en deux l'instance de locution, met simultanément en scène un locuteur a position fixe et un énonciateur à position variable. Il apparaît alors que si c'est toujours je qui parle ce n'est ni l'ici ni le maintenant du locuteur que l'énonciateur prend nécessairement comme repère. Loin d'être l'indice d'une fracture syntaxique dans l'architecture spatiotemporelle du discours, ce jeu énonciatif, qui s'appuie crucialement sur la mobilité référentielle des embrayeurs et des formes démonstratives ${ }^{1}$,

1. Relativité référentielle que mettent plaisamment à nu les personnages de Lewis Carroll dans Alice au pays des meroeilles, * le plus linguistique des ouvrages de fiction * selon l'expression de Claude Hagège : «-...l'archevêque patriote de Canterbury, Stigand lui-même, trouvant cela opportun... - Trouvant quoi ? demanda le Canard. - 
assure au sein de l'énoncé une parfaite cohésion entre les différentes chronologies mises en jeu par l'acte d'énonciation.

\section{Verbe et deixis.}

L'une des difficultés auxquelles se voit invariablement confrontée toute personne s'initiant a l'étude d'une langue étrangère est celle de l'acquisition des mécanismes lui permettant de concevoir (donc de s'approprier) la représentation exacte que le locuteur dit nat if de la langue dont il fait l'apprentissage se donne de l'espace et de son partage dans le système. Ces difficultés, si l'on s'en tient a la description des moyens déictiques du français et de l'espagnol, sont dues aux dissymétries que présentent, d'un point de vue comparatif, les deux systèmes, l'espagnol n'ayant pas atteint le degré d'abstraction auquel - comme l'a mis en évidence Maurice Molho ${ }^{2}$ - est parvenu le système français : là où l'espagnol s'est vu perpétuer, au terme de nombreux glissements et de réquuilibrages divers, un système ternaire (hic-iste-ille / este-ese-aquel), le français a évolué vers un système binaire qui ne distingue, dans le partage imaginaire de l'espace, que deux seules et uniques zones (celui-ci/celui-là). Cette dissymétrie se fait tout naturellement sentir dans la pratique de cet exercice éminemment comparatif qu'est la traduction, et ce, de manière d'autant plus patente que, de binaire, le système français de la représentation spatiale devient unitaire lorsque au déictique ce n'est pas joint l'un des deux clitiques discriminateurs $-c i /-l a ̀$. La construction du systeme français des déictiques déclinables - explique Maurice Molho - « repose sur la dissociation, fondamentale en pensée française, de la deixis spatiale et de la personne, ce dont résulte un morphème qui permet de situer sans référence aucune au moi pensant ou au non-moi : "ce chat", "cette maison", "ces enfants", s'ils ne sont accompagnes d'un geste ou d'une référence strictement discursive, ne disent nullement une position, mais seulement que l'être dont il est parlé a son lieu dans l'espace ${ }^{3}$ ».

Trouvant cela, répondit la Souris d'un ton contrarié. Bien sûr, vous savez ce que cela veut dire. - Je sais assez bien ce que cela veut dire quand c'est moi qui trouve quelque chose, repartit le Canard : c'est en général une grenouille, ou un ver. La question est de savoir ce que trouva l'archevêque » (III, p. 57).

2. * Linguistique et grammaire. Remarques sur le système des mots démonstratifs en espagnol et en français " in Linguistique et langage, Editions Ducros, 1969, p. 103-137. Cf. également Paulo de Carvalho, * "Deixis objective" v. "deixis subjective". Sur les "substantifs démonstratifs" du latin " in Bulletin de la Société de Linguistique de Paris, LXXXVI, 1, 1991, p. 211-244.

3. Ibidem, p. 134. 
Il s'ensuit qu'à un seul et même signifiant dans le système français (ce) peuvent théoriquement correspondre, dans le système espagnol, trois signifiants différents (este / ese / aquel), parmi lesquels le traducteur devra nécessairement opérer un choix. C'est là un aspect qui retient largement l'attention des auteurs de manuels scolaires et de guides de traduction, qui multiplient les notes et les commentaires relatifs à la traduction des formes déictiques. Malgré les difficultés qui résultent de ces déséquilibres entre les deux systèmes, la "règle " que prescrivent les grammaires normatives et pédagogiques ne semble pourtant pas poser de difficulté majeure : dans le cadre de ce qu'il est traditionnellement convenu d'appeler la deixis "situationnelle», un verbe dont le temps déclare l'inactualité passée ou future du procès envisagé a pour effet de ne faire admettre dans l'énoncé où il apparaît que des formes démonstratives susceptibles de déclarer l'«éloignement " de l'objet de référence par rapport au présent de parole de l'énonciateur, le déictique este, ancré dans son présent de locution, étant de ce fait rigoureusement exclu. Salvador Fernández Ramírez énonce cette « loi » comme suit :

...se dibuja vigorosamente con el uso de aquel una deixis más concreta y directa, frente a la deixis de este, que es rigurosamente textual. He aquí las condiciones. Aquel se refiere con notable regularidad $1^{\circ}$ a pasajes $o$ momentos $[\ldots] ; 2^{\circ}$ a palabras reproducidas en discurso directo $[\ldots] ; 3^{\circ}$ a hechos, actos, incidencias y situaciones singulares $[. ..] ; 4^{\circ}$ a estados, situaciones, procesos $o$ cualidades duraderas [...] Es curioso el hecho de que en estos usos [...] el empleo del pronombre " aquel" oa asociado al empleo predominante del pretérito. El demostratioo "este" puede decirse que está excluido de los tres primeros usos enumerados.

(Gramática española, §132)

Le principe est formulé dans des termes analogues dans la plupart des grammaires pédagogiques conçues à l'attention des étrangers qui, tout en mettant l'accent sur la "richesse " du paradigme déictique espagnol, insistent sur les incompatibilités qui président à la combinatoire syntagmatique des temps et des formes démonstratives à l'intérieur de l'énoncé. Les grammaires d'Andrés Bello, de Jean Bouzet et de J. Coste et A. Redondo, pour ne citer que celles-ci, soulignent cet aspect de la morphosyntaxe de l'espagnol :

Otras veces la demostración recae sobre el tiempo, y este, esto, señalan lo presente : aquel, aquello, lo pasado o lo futuro. Así esta semana es la semana en que estamos; aquel año es ordinariamente un año tiempo ha pasado.

(Andrés Bello, Gramática de la lengua castellana, § 257, p. 67)

Ces trois démonstratifs correspondent à une graduation d'éloignement dans l'esprit de celui qui parle, este désignant toujours le terme le plus rapproché, aquel, le plus éloigné, et ese, une position intermédiaire ou bien indifférente. a) 
Dans l'espace [...] b) Dans le temps : «Esta mañana ", ce matin (d'aujourd'hui); * aquella mañana ", ce matin-là (d'un autre jour).

(Jean Bouzet, Grammaire espagnole, § 108, p. 56)

L'emploi des adverbes démonstratifs de la première zone n'est possible que dans un contexte au présent, à moins qu'il y ait identité de lieu entre le passé (ou le futur) et le présent.

(J. Coste, A. Redondo, Syntaxe de l'espagnol moderne, p. 228)

Mais il n'est pas rare de voir apparaître ce même principe dans des études proprement linguistiques. Vidal Lamíquiz, par exemple, fait de cette règle de la concordance déictico-verbale un principe absolu :

La mostración temporal, precisamente por ser tiempo, implica que el pasado sea anafórico y que el futuro sea catafórico. Ambos, en una u otra dirección, están alejados y ausentes del hablante y del oyente [...] La señalización en el pasado y en el futuro temporales se oponen en uno y otro sentido con el presente. Este presente es siempre único y ad oculos [...] Se habrá deducido fácilmente la correspondencia que existe entre el verbo y el demostrativo de un mismo sintagma [...] Ambos señalan el momento (tiempo) en que se desarrolla la idea. Su relación es lógica y necesaria. Así, a un imperfecto corresponderá un demostrativo anafórico [= ese, aquel], a un futuro un catafórico [= ese, aquel], a un presente una deixis ad oculos $\left[=\right.$ este $^{4}$.

De même Emilio Alarcos Llorach affirme :

Lo que se incluye en el círculo de intereses coetáneos del hablante se indica con este, lo que pertenece a la esfera del pasado se introduce con aquel. Compárese, por ejemplo, i Cuántos oiajes hace este Kissinger! con i Qué cosas tenía aquel Jrusof $!^{5}$.

Ajoutons enfin que les déictiques dits "temporels" (ahora / entonces, hoy / aquel dia, etc.) sont, toujours selon la grammaire traditionnelle, soumis aux mêmes conditions d'emploi que leurs correspondants dits "spatiaux». Dans une autre étude, Emilio Alarcos Llorach explique à ce sujet :

Se emplea el perfecto compuesto con los adverbios que indican que la acción se ha efectuado en un período de tiempo en el que se halla comprendido el momento presente del que habla o escribe : hoy, ahora, estos dias, esta semana, esta tarde [...] Hay, no obstante, que señalar que con esta mañana, antes, se puede emplear el perfecto simple cuando las dos expresiones temporales citadas se sienten como oposición a esta tarde, ahora $[\ldots] 2^{\circ}$ Se emplea el perfecto simple con los adverbios que indican que la acción se produce en un

4. * El demostrativo en español y en francés. Estudio comparativo y estructuración $»$ in Reoista de Filología Española, Madrid, CSIC, 1967, p. 187-188.

5. « Los demostrativos en español " in Estudios de gramática funcional del español, Madrid, Gredos, 1970, § 14. 
período en el que no está incluido el momento presente del que habla : ayer, anoche, el mes pasado, aquel día, un día, hace años, entonces, cuando, etc. ${ }^{6}$

\section{Pour une " grammaire des fautes».}

\section{Soit l'énoncé :}

...cette femme méchante lui fit l'effet d'une pierre de plus que l'on trouve dans un chemin de montagne.

(Stendhal, Lucien Leuwen, XVI)

Confronté à la traduction de ce passage, un étudiant d'espagnol qui choisirait de rendre le syntagme cette femme par esta mujer se verrait probablement reprocher une "faute " de concordance déictico-verbale : s'agissant d'un récit au passé simple et d'un démonstratif opérant, on peut le supposer, une deixis non contextuelle, le démonstratif esta, indissolublement attaché au présent spatio-temporel du Moi, aurait pour effet, si l'on s'en tient aux explications grammaticales évoquées jusqu'ici, de détruire la cohérence temporelle du discours. Autrement dit, l'actualisation du déictique esta serait aussi « discordante » ici que pourrait l'être, dans ce même contexte, celle d'un adverbe d'actualité comme ahora accompagnant un verbe au prétérit. Peut-ĉtre est-ce pour éviter une telle distorsion que Consuelo Berges a rejeté, dans sa traduction du roman de Stendhal, le déictique esta au bénéfice du démonstratif de troisième degré : « ...aquella malévola mujer le hacía el efecto de una piedra más de las que se encuentran en un mal sendero en las montañas ". Que l'on considère cependant l'environnement verbal du démonstratif ce et l'on s'apercevra que la distance temporelle qui, instaurée par le prétérit fit, sépare l'événement narré du présent de la narration, est abolie par le déictique temporel aujourd'hui qui, réputé inapte à dater des événements passés, apparaît à l'ouverture de l'énoncé. En effet, s'il existe un contexte qui autorise à "enfreindre " le principe de la concordance déictico-verbale, c'est bien celui dont nous avons artificiellement isolé la proposition donnée en exemple, que nous retranscrivons maintenant dans son intégralité :

Lucien fut heureux de trouver madame de Serpierre bien ridicule. Un quart d'heure plus tôt, il eût ri de grand coeur ; maintenant cette femme méchante lui fit l'effet d'une pierre de plus que l'on trouve dans un chemin de montagne.

A Luciano le complació mucho encontrar muy ridícula a madame de Serpierre. Un cuarto de hora antes se hubiera reído de la mejor gana; ahora

6. « Perfecto simple y perfecto compuesto ", ibidem, p. 24-25. 
aquella malévola mujer le hacía el efecto de una piedra más de las que se encuentran en un mal sendero en las montañas.

L'emploi du démonstratif aquella n'a bien sûr rien de répréhensible du point de vue grammatical. Notre propos n'est d'ailleurs pas de discuter les choix opérés par C. Berges dans la traduction de ce passage, même s'il est possible d'y déceler plusieurs exemples de ce que Jean Claude Chevalier et Marie France Delport ont appelé "figures de traduction ${ }^{7}$ " - transformations que le système linguistique d'arrivée n'impose pas -, à commencer par l'altération du temps verbal qui de prétérit dans le texte source (fit) devient dans le texte d'arrivée un imparfait (hacia). Nous nous contenterons de faire remarquer ici que l'actualisation du démonstratif esta accompagné d'un verbe au passé combinaison habituellement tenue pour incorrecte - n'aurait pas été plus « agrammaticale » dans la traduction espagnole que ne l'est dans l'original français la combinaison "aujourd'hui + prétérit ». Souvent posé en termes d'intégration et de cohérence syntactico-sémantiques, le problème de la concordance déictico-verbale ne se laisse pas réduire, on le voit bien, a une simple question de grammaticalité : les deux possibilités (esta / aquella) s'offrent au traducteur et aucune des deux ne saurait être tenue pour " incorrecte " ou pour plus « correcte " que l'autre. Le choix du déictique dépend uniquement ici de la représentation que le traducteur, qui est avant tout un lecteur, voudra se donner de la position occupée par l'énonciateur à ce moment précis de la narration, position ambiguë puisque doublement indexée par le prétérit fit, qui situe le narrateur dans son présent de parole - ce qui l'amène à appréhender les événements de la fiction comme des événements passés -, et par l'adverbe aujourd'hui, qui, l'arrachant à son présent de narration, le situe dans le passé de l'univers fictionnel - ce qui lui permet de concevoir les événements narrés comme des événements présents -. Comme par ricochet, c'est le lecteur lui-même qui se voit conférer le pouvoir d'échapper imaginairement à son présent de lecture, l'adverbe aujourd'hui faisant naître chez lui

7. Voir notamment Jean Claude Chevalier * Traduction, littéralité et chaîne de causalités » et Marie France Delport * Traduction et littéralité : de la subjectivité dans les traductions de Madame Booary" in Littéralité 2. L'esprit de la lettre, Presses Universitaires de Bordeaux-Maison des Pays Ibériques, 1992, p. 45-78, ainsi que les travaux des deux auteurs parus dans La traduction. Actes du XXIII congrès de la Société des Hispanistes Français, Centre de publications de l'Université de Caen, 1989, p. 3-33 et 89-105, respectivement.

8. On consultera avec profit le numéro 104 de la revue Langages, 1991, consacré à l'ntégration syntaxique et à la cohérence discursive. Cf. en particulier Jacques Bres (« Le temps, outil de cohésion : deux ou trois choses que je sais de lui $m, p .92-110$ ), qui emprunte à Guillaume, en les redéfinissant, les concepts $d$ '* ascendance » et de * descendance » et les applique à l'analyse de l'ordre séquentiel des propositions narratives. 
l'illusion qu'il est a la fois contemporain du narrateur et des personnages de la narration. Situé entre ces deux marqueurs spatiotemporels, le démonstratif $c e$ se laisse alors interpréter tantôt comme une marque anticipée du retour au passé, temps qui sera rétabli un peu plus loin par le prétérit fit, tantôt comme un prolongement du présent imaginaire instauré par le déictique aujourd'hui, c'est-à-dire comme un actual-isateur, aux deux sens linguistique et étymologique du terme, chargé tout à la fois de déterminer le nom et de le présentifier imaginairement, c'est-à-dire de le rendre présent à l'esprit du locuteur. Que le narrateur échappe à son présent de parole pour se situer imaginairement dans son passé de narration comme s'il était contemporain des personnages ou qu'au contraire il les attire fictivement a son présent de locution, la stratégie énonciative reste identique dans les deux cas qui vise à abolir l'écart qui sépare le passé fictionnel du récit toujours présent qui en est fait par le narrateur.

On pourrait croire cependant que ce type de combinaison de morphèmes temporels en apparence incompatibles à l'intérieur d'un même énoncé constitue un simple accident grammatical, une bizarrerie morphosyntaxique isolée qui, du fait même de sa marginalité, ne saurait invalider dans le principe les postulats traditionnels de la concordance déictico-verbale. Mais le phénomène est loin d'être marginal ainsi que Marcel Vuillaume, à qui nous devons l'exemple cité ci-dessus, l'a récemment mis en évidence pour le roman français moderne et contemporain dans une étude importante sur la temporalité du récit'. Ces emplois qui, isolés de leur contexte, peuvent surprendre un lecteur qui pourtant ne les remarquera même pas, nombreux soient-ils, en lisant l'ensemble du roman, n'ont en effet rien d'exceptionnel. Massivement attestées dans le roman espagnol et hispanoaméricain, les combinaisons de formes déictiques comme ahora ou hoy suivies de verbes au prétérit, à l'imparfait ou au plus-queparfait, dénoncent un transport de voix qui met simultanément en cause deux temps totalement différents : le temps événementiel et le temps d'énonciation. En voici quelques exemples :

«AHORA » + IMPARFAIT :

Ahora, gracias a Dios, estaba Silda en puerto seguro, y el Cabildo había puesto en los casos a las deslenguadas sin vergüenza...

(José María de Pereda, Sotileza, IX, p. 70)

9. Grammaire temporelle des récits, Paris, Les Editions de Minuit, 1990, p. 31 et s. 
Ahora mismo (qué palabra, ahora, qué estúpida mentira) podía ${ }^{10}$ quedarme sentado en el pretil sobre el río, mirando pasar las pinazas negras y rojas...

(Julio Cortázar, Las babas del diablo, p. 208)

Esta biografía era la del chico y la de cualquier chico, pero a éste lo veía ahora aislado, vuelto único por la presencia de la mujer rubia que seguía hablándole.

(Julio Cortázar, Id., p. 210)

Primero entraba la mujer, recelosa ; ahora llegaba el amante lastimada la cara por el chicotazo de una rama.

(Julio Cortázar, Continuidad de los parques, p. 8)

...intenté asirme desesperadamente a los bordes de la verdadera circunstancia en que despertaba. Sólo que ahora la realidad era todavía peor, como si estuviera despertando a una pesadilla al revés.

(Ernesto Sábato, Sobre héroes y tumbas, III, XXXV, p. 429)

«AHORA »+ PRÉTÉRIT :

Otra vez se miraron. Ahora sonrieron los dos. Ana se levantó cinco minutos después.

(Leopoldo Alas Clarín, La Regenta, XXII, p. 503)

Ahora tres himnos se alojaron en mi pecho y ardían de modo inmarcesible, como lámpara de tres lenguas iguales...

(Ramón Pérez de Ayala, Belarmino y Apolonio, VII, p. 267)

Al abrir los ojos (tal vez gritaba ya) vio que se habían separado. Ahora sí gritó. De frío, porque la nieve le estaba entrando en los zapatos.

(Julio Cortázar, Lejana, p. 98)

...pero el Azarías ni le miraba, tan sólo sostenía la cuerda, cuyo cabo amarró ahora al camal en que se sentaba y se frotó una mano con otra...

(Miguel Delibes, Los santos inocentes, VI, p. 175)

Siguiendo ahora el borde de la cama, siempre hacia la derecha, encontré primero la mesita de luz y de nuevo la pared desconchada. Ahora estaba seguro : la respiración se hacía más nítida...

(Ernesto Sábato, Id., IV, III, p. 488)

10. Cf. * Así que ahora, en ese presente prematuro (como si el tiempo se divirtiese en presentarse antes de lo debido [...]), en ese presente que debería ser futuro, entraba falsamente su padre, subía aquellas escaleras que durante tantos años no había transitado " (Ernesto Sábato, Sobre héroes y tumbas, I, VII, p. 42). 
«HOY » + IMPARFAIT :

La rara aventura que hoy empezaba no era de las que podían emprenderse con mujeres en un buque donde, desde ahora, quien tuviera empeño de asearse tenía que hacerlo a la vista de todos...

(Alejo Carpentier, El siglo de las luces, XI, p. 166)

«AHORA » + PLUS-QUE-PARFAIT :

Ahora los franceses se habían adueñado del Comité de Bayona, eliminando a los españoles...

(Alejo Carpentier, Id., XIV, p. 186)

Volví, pues, al taller. Ahora que lo habia decidido, me empujaba una especie de desaforada ansiedad.

(Ernesto Sábato, Id., III, XXIX, p. 408)

Quelle est la portée syntaxique des adverbes hoy et ahora dans chacun de ces énoncés? Quel type de repérage temporel y construisent-ils? Que l'adverbe de temps n'est pas ici, comme le prétendent les grammaires scolaires a la lumière de l'étymologie du terme, un simple complément du verbe ou, comme on le dit parfois, une expansion de celui-ci, voila qui ne nécessite aucune démonstration : un signifiant adverbial qui dit l'actualité ne saurait uniquement porter sur un signifiant verbal qui déclare l'inactualité. Si, d'autre part, la notion d'« adverbe de phrase » semble mieux convenir a la caractérisation de ce type d'emploi, elle ne rend pas compte de la portée réelle de l'adverbe, qui, selon la définition du terme, est supposé modifier l'ensemble de la phrase sans porter sur aucun de ses constituants en particulier. Le probleme se laisse mieux cerner, me semble-t-il, dans le cadre de la théorie guillaumienne, qui fait de l'adverbe - et de tout constituant, synthétique ou analytique, assimilé à cette fonction - la seule catégorie du nom à ne pouvoir porter directement sur un segment précis de la phrase, le propre du signifiant adverbial étant de modifier le rapport existant entre deux segments distincts de la proposition. En effet, tout adverbe implique nécessairement deux éléments eux-mêmes reliés par un rapport d'incidence préconstruit que celui-ci ne vient compléter que dans un deuxième temps. S'il m'était permis d'élargir cette définition ou, plus exactement, de la rapprocher des vues de la théorie de l'énonciation, je dirais, partant du principe que tout adverbe est incident à un rapport d'incidence, que les deux unités ici en cause ne sont pas deux éléments du discours, mais deux vecteurs de l'énonciation, à savoir : l'énoncé, d'une part, et l'énonciateur, d'autre part. Autrement dit, tous ces déictiques temporels font office de modalisateurs adverbiaux incidents non pas aux énoncés où ils apparaissent, mais à l'énonciation dont ils résultent ou, pour mieux dire, au rapport modal institué entre le 
producteur de l'énoncé et l'objet de l'énonciation. Il s'ensuit que les adverbes hoy et ahora n'ont pas pour fonction de dater ici les événements que déclarent les verbes qu'ils accompagnent, mais le procès actuel de leur énonciation et de leur prise en charge par le locuteur, qui inscrit non pas les événements passés dans un présent imaginaire, mais son propre présent d'énonciation dans le temps de l'histoire qu'il rapporte. Dédoublé, l'énonciateur polyphonique que mettent en scène tous ces énoncés mène de front deux récits simultanés : celui de l'histoire et celui de sa narration.

Tous ces emplois témoignent à la fois du pouvoir $d u$ locuteur à échapper à son présent d'univers - il feint ainsi d'être contemporain des êtres dont il parle, de voir se dérouler sous ses yeux des événements qui sont, au moment où il les évoque, entièrement révolus - et de son impuissance à se soustraire à son présent d'énonciation, car en voyageant imaginairement dans le temps, en se projetant mentalement dans une époque inactuelle qui n'est pas celle où siège effectivement le sujet parlant, il ne fait jamais que passer d'un présent à l'autre : du présent, révolu ou à venir, qu'il évoque comme étant le théâtre des événements, à celui qu'il occupe en tant qu'énonciateur se donnant hic et nunc une représentation directe de l'événement en question, c'est-àdire d'un présent événementiel à une concevabilité présente de ce même événement. C'est à l'emprise de ce présent d'énonciation auquel nul locuteur n'est, quoi qu'il fasse, libre de se soustraire que saint Augustin fait allusion lorsque, récusant la division traditionnelle du temps en trois époques prétendument autonomes, il fait du présent le seul lieu possible de la représentation de l'image du temps :

Ce qui maintenant m'apparaît comme clair et transparent, c'est que ni le futur, ni le passé, ne sont. C'est donc improprement que l'on dit : « Il y a trois temps, le passé, le présent et le futur». Plus exactement dirait-on peut-être : * Il y a trois temps : le présent du passé, le présent du présent, le présent du futur ". Ces trois modes sont dans notre esprit, et je ne les vois point ailleurs. Le présent des choses passées, c'est la mémoire; le présent des choses présentes, c'est la vision directe ; le présent des choses futures, c'est l'attente ${ }^{11}$ ».

(Confessions, 11, 26)

11. * Quod autem nunc liquet et claret, nec futura sunt nec praeterita, nec proprie dicitur : "tempora sunt tria, praeteritum, praesens et futurum", sed fortasse diceretur: "tempora sunt tria, praesens de praeteritis, praesens de praesentibus, praesens de futuris". Sunt enim haec in anima tria quaedam et alibi ea non video, praesens de praeteritis memoria, praesens de praesentibus contuitus, praesens de futuris expectatio (trad. de Pierre de Labriolle). Je dois cette citation à Paulo de Carvalho que je remercie pour ses suggestions et ses remarques stimulantes. 
Puisque la mémoire n'est rien d'autre que le présent des choses passées, il suffit que les vues de l'énonciateur soient momentanément détournées du passé dont il a ou dont il feint d'avoir la mémoire vers le procès actuel de sa reconstitution mentale, pour qu'émergent, instituant une chronologie d'intellection distincte de celle, événementielle, qui, par exemple dans le cas du récit, sert de support à la diégèse, des formes déictiques ancrées dans le présent spatio-temporel du Moi parlant. Un deuxième niveau de temporalité12 centré sur le procès d'énonciation lui-même s'instaure alors qui, construit en marge du temps événementiel et non soumis par conséquent aux règles mécaniques de la consecutio temporum ou à celles de la concordance déictico-verbale, marque de son empreinte la configuration déicticotemporelle de l'énoncé : au lecteur d'intégrer désormais à son présent de lecture ces deux strates de temps en fonction de sa propre expérience temporelle.

Entre ces deux chronologies, toutes sortes d'interférences et de collisions sont dès lors rendues possibles ${ }^{13}$, l'attraction exercée par le présent d'énonciation sur le passé événementiel pouvant entraîner, dans certains cas, l'assimilation complete de ces deux niveaux temporels. C'est ce qu'illustre la nouvelle de Julio Cortázar Reunión, dans laquelle le narrateur témoigne volontiers, après avoir choisi le passé pour inaugurer sa narration, de son impuissance a poursuivre dans ce temps le récit des événements, ce qui l'amènera à raconter une

12. * ...l'acte configurant qui préside à la mise en intrigue - explique Paul Ricœur - est un acte judicatoire, consistant à prendre ensemble ; [...] raconter c'est déjà "réfléchir sur" les événements racontés. A ce titre, le "prendre ensemble" narratif comporte la capacité de se distancer de sa propre production, et par là de se redoubler [...] Or, c'est à la faveur d'un tel déplacement de l'attention de l'énoncé narratif sur l'énonciation que les traits proprement fictifs du temps narratif prennent un relief distinct. Il sont en quelque sorte libérés par le jeu entre les divers niveaux temporels issus de la réflexivité de l'acte configurant lui-même " (Temps et récit II. La configuration dans le récit de fiction, Paris, Editions du Seuil, 1984, p. 92).

13. Poussée à son comble, l'interférence temporelle peut, dans le discours littéraire, générer des associations insolites de temps verbaux et de déictiques théoriquement incompatibles : Esto lo estoy tocando mañana [...] Esto ya lo toque mañana, es horrible, Miles, esto ya lo toqué mañana, déclare Johny, le saxophoniste schizophrène de El perseguidor de J. Cortázar. Le poète César Vallejo va encore plus loin lorsque, associant à un verbe conjugué au prétérit un déictique prospectif, il ouvre le sixième poème de Trilce par le vers El traje que oestí mañana : la rupture oestí / mañana entraîne la scission d'un présent refoulé par l'écriture, clivage temporel que le texte spatialise au vers 7 ( $A$ hora que no hay quien oaya a las aguas) en dissociant typographiquement le déictique d'actualité en deux mots, $a+$ hora, conformément à son étymologie (cf. notre étude * Transtextualidad en la escritura de César Vallejo. "En mis falsillas encañona..." : del étimo verbal al étimo textual $n$ in Homenaje Mundial a César Vallejo, Lima, Carlos Milla Batres Editor - sous presse -). 
partie de l'histoire au présent, un présent que l'on se gardera bien d'assimiler, malgré les affinités qui semblent l'y relier, au présent historique ${ }^{14}$ de la grammaire traditionnelle :

Aunque esto que cuento pasó hace rato, quedan pedazos y momentos tan recortados en la memoria que sólo se pueden decir en presente [...] Es la noche [...] Ahora hay que esperar otro amanecer... (p. 107)

La puissance de ce présent d'énonciation peut, analoguement, amener l'énonciateur à révoquer les règles de la concordance des temps, phénomène dont l'ampleur en espagnol moderne invite moins à parler de transgresion des principes de la concordantia temporum qu'à réfléchir sur les mécanismes énonciatifs qui permettraient d'élaborer, inversement, une théorie générale de la discordantia temporum. Condamnée par la norme ${ }^{15}$ mais largement attestée dans la langue actuelle, la non-concordance des temps, que l'on présente habituellement comme une « faute " de syntaxe ou, dans le meilleur des cas, comme une concordance ad sensum ou encore comme l'effet d'une attraction temporelle, met a jour une autre forme de cohésion énonciative que celle, strictement morphosyntaxique, que préconisent les grammaires normatives. En voici quelques exemples journalistiques ${ }^{16}$ :

14. Au sujet de ce présent dit « historique » Maurice Molho fait remarquer à juste titre que l'irruption dans un contexte où domine le passé (incident ou décadent) d'un verbe au présent permet de perspectiver les événements en instituant entre eux une hiérarchie interne : « Así, pues, un presente histórico o narrativo no es sino un presente de posición, cuya posición (sic) se utiliza en el discurso a fin de jerarquizar los acontecimientos del relato : sólo es expresivo por la jerarquía que instituye " (Sistemática del oerbo español, Madrid, Gredos, 1975, p. 248).

15. C'est cette approche éminemment normative qui, par exemple, amène Emilio Alarcos Llorach à affirmer : * la consecutio temporum decide el empleo de un tiempo determinado, pero no el que ese tiempo sea simple o compuesto [...] El nexo primero no exige un aspecto determinado en el nexo siguiente, pues podemos decir era posible que llegase, y era posible que hubiese llegado; pero sí exige un tiempo determinado, pues no podemos decir era posible que llegue " (Gramática estructural, Madrid, Gredos, 1977, p. 122). Beaucoup plus prudent, Maurice Grevisse explique à ce propos : « Sans doute, dans bien des cas, une concordance s'établit, qui règle le temps de la subordonnée par rapport au temps du verbe principal, mais bien souvent aussi il faut tenir compte de certaines modalités de la pensée, et marquer, selon une syntaxe appropriée, le temps de la subordonnée par rapport au moment où l'on parle : ainsi, par discordance des temps, peuvent être rendues bien des nuances délicates " (Le bon usage, §2728 - c'est moi qui souligne - ).

16. In Julia Ponsard, La non concordance des temps dans les phrases subordonnées en espagnol contemporain (mémoire de maîtrise inédit), Université de Bordeaux III, 1992. 
El escritor pidió el domingo al arzobispo de Canterbury que intervenga directamente ante los líderes religiosos de Irán para que pongan fin a la pena de muerte que pesa sobre él.

(El País, 26-11-1991)

Se quejó de que no se confirmen las noticias y calificó las relaciones actuales entre esos medios de * lamentables ».

(Id., 29-1-1992)

Etxabe advirtió a los abogados que, cuando salga de la prisión, al que le haga esas acusaciones, se lo llevará por delante.

(Id., 8-5-1992)

El secretario general [...] sugirió que sea la propia Comunidad Europea la que destaque una fuerza militar de mediación en esa región báltica.

(Id., 15-5-1992)

De tels emplois trouvent aisément explication dans le modele polyphonique proposé plus haut : les événements dont il est question dans tous ces énoncés sont l'objet d'une double mise en perspective temporelle qui amène l'énonciateur à adopter successivement deux points de vue différents : le sien et celui du locuteur dont il reprend les propos. Or ce transport temporel de la voix énonciative est un fait secondaire par rapport au jeu modal qu'il permet d'instaurer dans le discours. En effet, entre : 1. "Etxabe advirtió a los abogados que, cuando salga de la prisión, al que le haga esas acusaciones, se lo llevará por delante " et 2. "Etxabe advirtió a los abogados que, cuando saliera de la prisión, al que le hiciera esas acusaciones, se lo llevaría por delante ", il y va de bien plus que d'une simple question d'acceptabilité syntaxique, l'énoncé qui est conforme aux règles de la concordance des temps apparaissant, au point de vue énonciatif, comme une forme modalement atténuée par rapport à l'énoncé « agrammatical ». Si l'on a l'impression que l'énoncé de départ (" cuando lyol salga de prisión, al que me haga esas acusaciones, me lo llevaré por delante") perd de sa force illocutoire dans sa reprise en 2, c'est parce que la régression temporelle exigée par la concordance des temps (salga AE saliera, haga $A E$ hiciera, llevaré $A E$ llevaría) a pour effet de subsumer la modalité exercitive de l'énoncé de départ sous une modalité expositive qui la virtualise au point de transformer un énoncé primitivement admonitif en une assertion purement déclarative. C'est cette perte modale que la non-concordance des temps vise à compenser : en récusant la régression temporelle que réclame le discours rapporté, l'énonciateur de 1 incorpore dans son énoncé une modalité d'énonciation que l'énonciateur de 2 évacue complètement : les deux locuteurs reprennent un énoncé qui est étranger à leur propre discours, mais seul 
le premier recrée en outre les conditions d'énonciation et le contexte modal dans lesquels cet énoncé a effectivement été proféré.

Il apparaît donc que l'expression linguistique du temps et de l'espace ne renvoie, en dernière analyse, à rien d'autre qu'à l'énonciateur et à sa façon d'organiser l'énoncé. En effet les morphèmes spatio-temporels ne renseignent pas l'interlocuteur sur la position qu'occupe l'objet évoqué par rapport à l'énonciateur, mais bien sur celle qu'occupe l'énonciateur par rapport a l'objet dont il parle. Autrement dit : les déictiques ne servent pas à "situer ", mais à " se situer " soi-même dans l'espace et dans le temps par rapport à des repères librement fixés par le sujet parlant. Faire appel à un déictique ce n'est pas, comme on le dit parfois, assigner à un référent donné une place dans l'espace ou dans le temps - si tel était le cas on pourrait mesurer en mètres ou en heures le champ de monstration désigné par chacun d'entre eux - : c'est, au contraire, se donner à soi-même la position spatiale et temporelle permettant la saisie et la mise en perspective de l'être dont il est parlé. De même, comme le fait remarquer Michel Picard, "les "temps" des verbes, leurs emplois réguliers ou originaux, renvoient le lecteur à du signifié, non à du référent. A des représentations du temps, a des attitudes mentales, non au temps vécu ${ }^{17}$ ".

On ne s'étonnera pas dès lors qu'un temps verbal comme le prétérit, dont le propre est de déclarer l'inactualité des événements révolus, puisse évoquer des événements chronologiquement postérieurs au moment de leur énonciation. Il suffit pour cela que le Moi regardant se projette fictivement dans un temps ultérieur à celui, futur, où surviendra l'événement envisagé pour que le procès verbal se laisse concevoir aussitôt comme un pré-futur, donc comme un vrai passé par rapport au lieu temporel occupé par le locuteur :

Quantos nunca nascicron e fueron engendrados, quantos almas ovieron, fueron vivificados, si los comieron aves o fueron ablentados, todos en aquel día allí serán juntados.

(Gonzalo de Berceo, Signos que aparecerán..., XXIII)

...goza cuello, cabello, labio y frente, antes que lo que fue en tu edad dorada oro, lilio, clavel, cristal luciente, no sólo en plata o víola troncada se ouelva, mas tú y ello juntamente en tierra, en humo, en polvo, en sombra, en nada.

(Luis de Góngora, Mientras por competir...)

17. Lire le temps, Paris, Les Editions de Minuit, 1989, p. 53. 
PEDRO ABUIN. - Y si se niega, ¿ qué procede?

EL VIEJO DE CURES. - Esperar una mudanza de su genio. Tú propones juntarnos para la rebeldía. i Así es ! Yo, para las mediaciones que transigen guerras. ¡Quién tuvo razón, lo diga el tiempo!

(Ramón del Valle-Inclán, Cara de Plata, I, 1, p. 496)

Qu'au contraire l'énonciateur se situe imaginairement dans un temps révolu, et il percevra immédiatement comme appartenant au futur des événements qui, ultérieurs à son présent d'observation, sont pourtant antérieurs à son présent de parole :

Las doce, había dicho el sereno, i ya era mañana !, es decir, ya era hoy ; dentro de ocho horas la Regenta estaría a sus pies confesando culpas que había olvidado el otro día.

(Leopoldo Alas Clarín, La Regenta, XV, p. 322)

La double instanciation à laquelle l'énonciation polyphonique soumet le discours a pour effet de dissocier ces deux activités - résultativement concomitantes, mais opérativement décalées - que tout acte de parole met en cause, a savoir : le « dire », auquel s'attache un présent de locution imposé par la situation d'énonciation, et le "voir", auquel s'attache un présent d'observation que le sujet énonciateur choisit librement de se donner. Les yeux s'émancipent de la voix : le locuteur parle ici et maintenant d'un événement que son double énonciatif choisit délibérement de regarder d'un autre lieu et d'un autre temps que celui qu'occupe le producteur physique de l'énoncé (ce n'est sans doute pas un hasard si l'anglais identifie phonétiquement le nom de la personne locutive et le signifiant qui désigne l'organe de la vue $\left.: I / e^{18} e^{18}\right)$. Déporté de son présent de parole, l'énonciateur parle au nom d'un je dont l'ici trouve son siege ailleurs. L'énonciateur pouvant se donner, pour évoquer un même et unique procès, des lieux d'observation différents - des lieux qui seront pour lui autant de concevabilités spatio-temporelles différentes -, l'événement envisagé peut, au cours de son énonciation, être l'objet d'une visée perspectivale multiple. C'est ainsi que, invité sur un plateau de télévision, l'animateur d'une émission radiophonique bien connue basée sur le principe du canular téléphonique, à la question "quelle imposture avez-vous prévu de faire demain ?", a pu répondre, l'émission du lendemain ayant été pré-enregistrée : "Demain matin, j'ai téléphoné à...». On aura vite fait de condamner un tel brassage de morphèmes syntagmatiquement incompatibles; le processus énonciatif reste, quant à lui, parfaitement cohérent : le locuteur parle maintenant

18. Cf. Frédéric Regard, « Polyphonie des voix narratives et autorité énonciative » in Poétique, num. 89, 1992, p. 48-49. 
d'un événement passé qui, différé jusqu'au lendemain, sera perçu comme présent par l'auditeur.

\section{3. « Dire », « voir » et « savoir ».}

On voit donc se dégager à travers tous ces emplois deux postures d'énonciation différentes - prospective l'une, régressive l'autre -, qui dépendent uniquement de la position spatio-temporelle assignée par le locuteur à son double énonciatif. En se situant en un lieu temporel postérieur à l'événement futur que le locuteur souhaite évoquer, l'énonciateur appréhende rétrospectivement l'avenir et valide ainsi l'antériorité d'un fait qui pour le locuteur est - tout autant que le fait lui-même - encore incertaine. En revanche, en se situant en un lieu temporel antérieur à l'événement passé que le locuteur souhaite évoquer, l'énonciateur, qui conçoit le passé comme un temps en cours de réalisation, exclut de son champ visuel la subséquence d'un fait qui pour le locuteur est supposée connue. En d'autres termes, ce que l'un fait semblant de voir, l'autre fait semblant de l'ignorer ${ }^{19}$. On voit combien ce jeu énonciatif pose le problème de la régulation de l'information par le sujet parlant, effet incontournable de la linéarité du signe qui dans le discours narratif, où il revêt des implications particulières, met constamment aux prises le «dire " et le «savoir», c'est-à-dire ce que sait ou feint de savoir le narrateur dès sa première intervention dans le roman et ce qu'il peut, veut ou doit en laisser transparaître à chaque point de son récit. Ce paradoxe sera formulé par Jean Pouillon en 1946 dans les termes suivants :

Si l'on écrit au passé, c'est, semble-t-il, qu'au moment où l'auteur écrit, les événements qu'il raconte sont terminés et qu'il en possède maintenant le sens.

19. En figure :

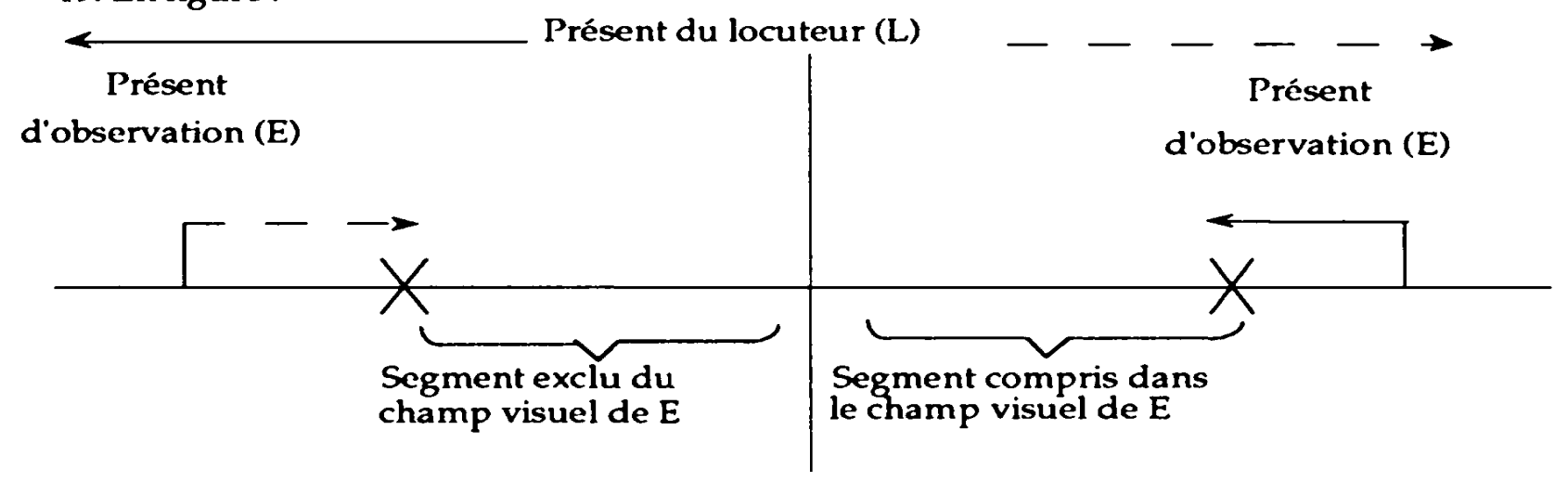


Alors comment peut-il rendre l'indétermination du présent de son héros, puisque ce présent est pour lui un passé ?20

C'est à ce problème technique que le discours indirect libre tente d'apporter une solution, en fournissant au narrateur un moyen efficace de restreindre ou, plus exactement, de contrôler le champ présuppositionnel que recouvrent les formes verbales.

La syntaxe de la condition offre à cet égard une possibilité d'analyse particulièrement éclairante. Si l'on compare les trois modèles que distinguent traditionnellement les grammaires descriptives - condition " réelle ", " irréelle » et "impossible " - on constate que, du point de vue de la présupposition, seules les conditionnelles dites "réelles" permettent d'asserter des faits indépendamment de toute réalisation. Ainsi, dire "si je pouvais " ou "si j'avais pu " revient immédiatement a affirmer "je ne peux pas » et " je n'ai pas pu ", a la différence d'une assertion comme "si je peux " qui pose un procès puissanciel dont il est impossible de déterminer s'il est validé ou non dans le présent et, à plus forte raison, s'il le sera dans l'avenir. Puisque l'avenir n'est incertain que pour autant que le présent ne l'a pas encore transformé en passé, le problème qui se pose dans le récit au passé est celui de la prospectivation au sein de ce temps d'événements qui sont à la fois contingents et révolus. En effet, comment faire pour évoquer au passé un fait hypothétique sans en faire immédiatement ressortir une subséquence que la forme du verbe déclarerait implicitement et que l'énonciateur est, par ailleurs, supposé connaître déjà ? Si le narrateur peut y parvenir, c'est justement en abandonnant son présent de locution et en installant son présent d'observation dans le temps où se situe l'action. On constate alors que là où le français ne discrimine pas modalement la condition "irréelle" de la condition " réelle " perspectivée (celle que le locuteur postule comme passée, mais que l'énonciateur conçoit comme présente), l'espagnol récuse pour cette dernière la régression modale (indicatif $A E$ subjonctif) que la première exige systématiquement. Ainsi, ce qui oppose un énoncé comme "si hiciera buen tiempo saldría ", qui déclare implicitement, par le seul choix dans la protase du mode subjonctif, l'impossibilité du procès à se réaliser $^{21}$ (= « no hace buen tiempo »), à « si hacía buen tiempo saldría ",

20.Temps et roman, Paris, Gallimard, 1946, p. 160 (cité par Michel Picard, op. cit., p. 73).

21. Jean Claude Chevalier, Michel Launay et Maurice Molho expliquent à propos de ce type de conditionnelle : * formular una hipótesis a contrario, es declarar que la experiencia se deja identificar bajo una forma opuesta a la que propone la aserción que por lo mismo es discutible » ( $\alpha$ Del morfema si $\gg$ in Philologica hispaniensa in honorem Manuel Aloar, II, Madrid, Gredos, 1985, p. 140). 
qui évacue toute espèce de présupposition, c'est que hiciera a comme repère temporel le présent du locuteur alors que hacía s'attache à un présent d'observation que l'énonciateur, détourné de son présent de parole, partage fictivement avec l'être dont il parle 22 :

Además, Ana, puesto que defendía su virtud, creía prudente ocultar todo lo que fueran personalidades al confesor. * Si crecía el peligro, hablaría. Mientras tanto, no".

(Leopoldo Alas Clarín, La Regenta, XIX, p. 403)

Si proseguían los desórdenes, iría a Pucrto Rico y luego a Surinam, sin detenerse en Haití [...] Si Dexter persistía en no hacer escala en Port-au-Prince, pronto oolverían allá las naves refugiadas en Santiago [...] Si la situación no era como la pintaban [...] Esteban regresaría por el mismo barco para buscar a su prima [...] Si la vida era posible en la isla, vendrían a buscarla.

(Alejo Carpentier, El siglo de las luces, I, XI, p. 159-161)

...el plazo definido y fijo lo hacía sentir de pronto dotado de un terrible poder y aumentaba su resentida satisfacción, como si se dijera ahora vamos a ver. Y si no se presentaba, se mataría [...] Además, si Dios se aparecía, i cómo lo haría ? [...] Y si se aparecía y él era incapaz de advertirlo? Entonces se mataría inútil y equivocadamente.

(Ernesto Sábato, Sobre héroes y tumbas, IV, V, p. 538-539)

C'est un tel rapport d'implication que le discours dit « indirect libre» permet d'évacuer par le dédoublement polyphonique de l'instance de narration en permettant à l'énonciateur de concevoir comme étant encore virtuels des événements qui pour le locuteur ne le sont manifestement plus. Il apparaît alors que ce qu'on appelle traditionnellement discours "indirect libre » et que l'on persiste à considérer comme un état $\mathrm{du}$ "discours de personnage ${ }^{23}$ » représente fondamentalement un état $d u$ "discours de narrateur » qui n'est ni forcément « libre " ni systématiquement «indirect». Il n'est pas libre puisqu'il est la stratégie obligée qu'adopte le narrateur pour exclure de son champ visuel - et donc pour ne pas laisser transparaître dans son discours - l'avenir médiat ou immédiat du passé qu'il feint d'occuper, c'est-à-dire pour tourner le dos à l'avenir d'un passé qui ne lui est pas inconnu (on comprend que l'imparfait, qui opere une saisie

22. C'est la raison pour laquelle on ne pourra, malgré l'absence de verbe dicendi, rendre par un subjonctif imparfait en espagnol l'imparfait de l'indicatif qui, en français, apparaît dans la protase des conditionnelles réelles perspectivées. Cf. le passage suivant : * En vérité cet amour de son enfant, elle ne l'avait jamais possédé : "J'ai renoncé à ce que je ne détenais pas ; j'ai fait le sacrifice de ce qui ne m'a jamais appartenu..." Tandis que si, demain soir, il lui fallait subir de Georges un nouvel assaut... Ah! cette fois, eh bien! elle mentirait... " (François Mauriac, La fin de la nuit, VI, p. 121).

23. Selon la terminologie de Genette (Figures III, Paris, Editions du Seuil, 1972, p. 191). 
régressivement vectorisée du procès, en laissant en suspens son segment final, constitue avec le conditionnel le temps par excellence de ce type d'énonciation). Indirect, il ne l'est pas non plus, puisque, bien que traditionnellement rangé, avec les discours direct et indirect, parmi les modalités du discours transposé, il n'opère que secondairement la reprise d'un discours antérieur. En effet, pour qu'il y ait rapport de discours il faut qu'il y ait un discours à rapporter : or, en interceptant in statu nascendi les pensées du personnage avant même que celui-ci n'ait pu leur donner la forme d'un vrai discours (déclaré ou intériorisé, réel ou virtuel), le narrateur, loin de rapporter un discours - qu'il ne peut transposer puisqu'il n'existe pas encore -, met lui-même en discours les pensées d'un personnage qui n'en a pas nécessairement, au moment où le narrateur s'en ampare, "une conscience verbalisée et réfléchie ${ }^{24}$ " (E. Faucher) : ce que rapporte le narrateur, si rapport il y a, ce ne sont pas les propos d'un personnage ( $\mathrm{du}$ moins ne le fait-il pas systématiquement), mais ceux de l'énonciateur fictif que le narrateur a non moins fictivement " dépêché " sur les lieux des événements. Aussi les effets d'immédiateté et d'immersion psychologique qui s'ensuivent sont-ils moins le résultat de l'intégration des pensées du personnage dans le discours du narrateur que celui de la visualisation prospectivée des événements à l'intérieur du passé, c'est-à-dire de l'instauration d'une sorte de présent au sein d'un temps qui est entièrement révolu.

\section{II. - « JE » EST UN AUTRE}

Le pouvoir de l'énonciateur à occuper simultanément des lieux spatio-temporels différents, s'il implique, quand il choisit de l'exercer, un dédoublement de son présent locutif, ne met pas en cause l'unité énonciative du sujet parlant, qui assume dans tous les cas la responsabilité des énoncés qu'il produit et cautionne le contenu de ses propos. En effet les énoncés qui ont été décrits jusqu'ici mettent en scène un seul et unique je qui, se situant en meme temps ici et ailleurs, focalise, en les mettant en perspective, les événements qu'il évoque. Autrement dit, malgré l'ubicuité de l'énonciateur, la source de l'énonciation demeure toujours unique et inchangée. Dans les énoncés dont nous entreprenons l'étude ici, c'est, au contraire, l'ici-maintenant du locuteur qui, sans subir aucun dédoublement, se trouve contradictoirement habité par deux voix différentes : celle du je qui parle et celle d'un autre qui investit momentanément sa parole. Ce jeu, qui génère dans le discours un effet de distanciation, constitue le

24. Définition du discours indirect, Université de Paris IV, 1978, p. 9 (cité par Marcel Vuillaume, op. cit., p. 107). 
fondement de ce que la rhétorique traditionnelle appelle l'ironie ${ }^{25}$, figure de pensée définie par Fontanier comme le trope consistant à « dire par une raillerie, ou plaisante, ou sérieuse, le contraire de ce que l'on pense, ou de ce que l'on veut faire penser ${ }^{26}$ ». Cette définition, dont nous tenterons, par un examen détaillé du phénomène, de montrer les limites, servira de fil conducteur à la deuxieme partie de notre analyse.

\section{1. «Dire par une raillerie, ou plaisante, ou sérieuse... "}

Cette première partie de la définition de Fontanier est représentative d'un certain mode de raisonnement qui consiste à juger le phénomène que l'on veut décrire non pas à l'opération qui le sous-tend, mais aux effets, extrêmement variables, que ses différentes réalisations peuvent susciter et donc à prendre le résultat du procès pour le procès lui-même ou, plus simplement encore, à confondre l'effet avec sa cause. Car définir l'ironie comme une forme de "raillerie" c'est - selon l'acception que l'on voudra retenir du terme - faire de l'ironiste un locuteur nécessairement malicieux, moqueur, sarcastique ou persifleur ${ }^{27}$. Certes, sous certaines de ses manifestations, l'ironie

25. Une bibliographie particulièrement dense sur le problème de l'ironie a vu le jour au cours des vingt dernières années. Outre les études que nous citons dans les pages qui suivent, on mentionnera ici les travaux de : Wayne $C$. Booth, A Rhetoric of Irony, Chicago, University of Chicago Press, 1974 ; M. Le Guern, \& Eléments pour une histoire de la notion d'ironie " in Catherine Kerbrat-Orecchioni et alii, Linguistique et sémiologie, II, Travaux du CRLS de l'Université de Lyon II, 1976, p. 53 et s. (réédition PUL, 1978); D. C. Muecke, Irony and the Ironic, London-New York, Methuen, 1980 ; John R. Searle, Sens et expression. Etudes de théorie des actes de langage, Paris, Les Editions de Minuit, 1982, p. 161 et s. ; Dell Hymes, compte rendu de l'étude de Dan Sperber et Deirdre Wilson (v. infra note 53) in Language in Society, 12, 1983, p. 100-103; B. Basire, * Ironie et métalangage " in DRLAV, num. 32, Université de Paris VII, 1985, p. 129-150; Dominique Maingueneau, Elements de linguistique pour le texte littéraire, Paris, Bordas, 1990, p. 69-83.

26. Les figures du discours, Paris, Flammarion, 1977, p. 146.

27. « [L'ironie] - poursuit Fontanier - semblerait appartenir plus particulièrement à la gaieté ; mais la colère et le mépris l'emploient aussi quelquefois, même avec avantage ; par conséquent, elle peut entrer dans le style noble et dans les sujets les plus graves " (p. 146). Pour Henri Bénac (Nouveau oocabulaire de la dissertation et des études littéraires, Paris, Hachette, 1972), devant un énoncé ironique, l'auditeur est « amené à comprendre qu'il y a chez ceux qui prétendent vraie la proposition, soit de la sottise, ce qui provoque sa raillerie, soit de la mauvaise foi, ce qui provoque son indignation » (p. 103). Analoguement, dans son Dictionnaire de poétique et de rhétorique (Paris, Presses Universitaires de France, 1966), Henri Morier explique : * Le ton de l'ironie est tantôt faussement enjoué, tantôt sifflant, emporté, cassant. C'est que l'ironie est commandée par un sentiment de colère, mêlé de mépris et du désir de blesser afin de se venger " (p. 217). Puis, à propos du passage des Provinciales où Pascal, se défendant des jésuites qui lui reprochaient de se servir de cette forme d'expression " peu charitable ", rappelle que Dieu lui-même avait eu recours à l'ironie, Morier ajoute : « L'ironie 
s'apparente à la raillerie, mais postuler une équivalence rigoureuse entre les deux termes c'est faire de ce qui n'est qu'un effet - ou parfois meme une impression - un principe explicateur.

Consciente de la multiplicité de formes que peut adopter l'ironie, Maria Helena de Novais Paima, dans l'étude dense et documentée qu'elle consacre à ce trope, fait remarquer que le procès d'expression per contrarium " representa apenas uma particularização, uma manifestação típica, se se quiser, de uma atitude interior que se manifesta de muitas outras maneiras ${ }^{28} »$. Il reste, malgré cette déclaration de principe, que l'auteur s'attache essentiellement dans son essai à décrire et à répertorier les différents effets suscités par l'énoncé ironique, sans véritablement chercher à dégager un principe susceptible d'expliquer globalement l'ensemble de ses manifestations :

Se nela predomina uma feição de alegria amigável, individualiza-se em humor ; se traduz uma amargura ácida, chama-se então sarcasmo; se joga agudamente com conceitos, recebe o nome de espírito; se se alia ao burlesco, toma a forma de facécia; se recorre a imitação, diferença-se em sátira ${ }^{29}$.

Humour, sarcasme, esprit, facétie, satire..., autant de termes communément associés à l'ironie, pourtant inaptes a rendre compte des mécanismes qui en sont à l'origine.

Il est vrai que l'énoncé ironique se présente très souvent sous forme d'antiphrase railleuse et que le procédé est dans bon nombre de cas exploité à des fins plus ou moins humoristiques ${ }^{30}$. Mais définir l'ironie a partir de paramètres aussi diffus que l'intentionnalité (plus ou moins railleuse) du locuteur ou l'effet (plus ou moins comique) qu'elle suscite chez l'auditeur, c'est s'en tenir à des considérations tout à fait extérieures au phénomène. Aucun de ces deux aspects n'est, d'ailleurs, déterminant ni nécessaire pour caractériser l'ironie, qui peut, au demeurant, n'être ni railleuse, ni comique (ni même - on le verra plus loin - antiphrastique), comme le fait apparaître l'extrait du dialogue suivant :

s'accompagne d'un sentiment de parfaite supériorité ; c'est pourquoi elle convient à Dieu (sic) et c'est pourquoi elle comporte chez les hommes une nuance de dédain. Ironiser, c'est manquer de modestie " (p. 217). C'est moi qui souligne.

28. Contribuição para uma estilística da ironía, Lisboa, Publicações do Centro de Estudios Filológicos, 1961, p. 3.

29. Ibidem, p. 3

30. Sur ce point cf. * Humour et Ironie » in Bulletin du Centre d'Etudes et Discussions de Littérature Générale, VII, Université de Bordeaux III, 1957-1959. 
- ¡ Madre, madre, por Dios! Yo no soy un niño...

- No, no eres un niño ; a ti no te duele que tu madre se consuma de impaciencia, se muera de incertidumbre. La madre es un mueble que siroe para cuidar de la hacienda, como un perro; tu madre te da su sangre y se arranca los ojos por ti, se condena por ti, pero tú no eres un niño, y das tu sangre, y los ojos, y la salvación... por una mujerota.

(Leopoldo Alas Clarín, La Regenta, XV)

Qu'y a-t-il de comique dans la réplique de la mère? Est-ce « par une raillerie " qu'elle se compare ou, plus exactement, qu'elle identifie son rôle à celui d'un meuble ou à celui d'un chien? Pourtant le locuteur dit bien «le contraire » de ce qu'il "pense » et de ce qu'il «veut faire entendre ", et l'on ne saurait, sous peine de contresens, lire l'énoncé no eres un niño comme une expression litotique, dont le sens (*eres todo un hombre, par exemple) irait à l'encontre de celui que semble imposer le contexte énonciatif. A quoi on pourrait objecter que la mère ne fait que reprendre dans son discours les derniers mots qui ont été prononcés par son fils. Mais loin de constituer - comme l'ont affirmé certains auteurs ${ }^{31}$ a propos de cette modalité de reprise - une forme de discours rapporté, la réplique no eres un niño constitue la reprise intériorisée d'une phrase qui, formulée par un locuteur, est énoncée par une instance de parole différente de l'instance de locution qui la produit. En d'autres termes, le locuteur ironique - en l'occurrence la mère - est le producteur matériel d'un énoncé dont il n'assume pas l'énonciation. Il s'ensuit que le contenu de la phrase n'est pas pris en charge par le producteur empirique de l'énoncé qui, se dédoublant fictivement, décline toute responsabilité sur l'énonciateur imaginaire auquel, tel un ventriloque, il feint de donner la parole : par la bouche de la mère c'est un autre énonciateur qui parle. D'où le jeu polyphonique, discours à «deux voix » né de la scission énonciative de l'instance de

31. Telle est, par exemple, la position d'Ann Banfield ( Où l'épistémologie, le style et la grammaire rencontrent la théorie littéraire " in Langue française, 44, 1979, p. 9-26), qui voit dans ce type d'énoncé une forme de discours rapporté soumis à un verbe dicendi passé sous silence (par exemple : « [Dices que] no eres un niño »). Cette interprétation a été contestée par Oswald Ducrot qui a remis en question l'unicité prétendue du sujet parlant : * Lorsque le sens d'un énoncé comporte l'incontestable indication d'un locuteur (attestée par la présence de pronoms de première personne) et que cependant l'énoncé exprime un point de vue qui ne peut pas être identifié à celui du locuteur - par exemple, lorsque quelqu'un, ayant été traité d'imbécile, répond "Ah, je suis un imbécile, eh bien, tu vas voir..." - Banfield est obligée d'exclure ces "reprises" du champ du style indirect libre et d'en faire un des modes du discours rapporté (en décrivant le "je suis un imbécile" du discours précédent comme un "tu dis que je suis un imbécile"). Grâce à de telles exclusions, elle peut formuler un principe selon lequel, lorsqu'il y a un locuteur, celui-ci est nécessairement le sujet de conscience, principe qui n'a d'autre justification, à mes yeux, que de sauver une unicité admise a priori comme une donnée de bon sens : "On ne peut pas, dans un énoncé que lon présente comme le sien, exprimer un point de vue qui ne serait pas le sien" "( Le dire et le dit, Paris, Les Editions de Minuit, 1984, p. 172-3). 
locution, sous lequel se laissent entendre, ensemble mais en conflit, la voix du sujet qui " parle " et celle du sujet qui "énonce". Analoguement lorsque le personnage dit la madre es un mueble..., c'est bien lui qui « parle" mais ce n'est pas son point de vue qu'il exprime. Ce que traduit cette phrase c'est la position d'un énonciateur fictif qui, par ses propos outranciers, introduit une disproportion massive entre son propre point de vue et celui du locuteur qui lui sert de truchement énonciatif. La mère ne pense pas que son rôle doive consister à cuidar de la hacienda, como un perro, pas plus qu'elle ne reproche a son fils de l'avoir dit - ni même de l'avoir pensé - : elle fait comme si celui-ci l'avait effectivement dit et feint ainsi de reprendre un énoncé jusqu'alors inactualisé.

«L'ironie - explique Vladimir Jankélévitch - est une variété de l'allégorie, ou plutôt de la "pseudégorie"... mais [...] cette mendacité, l'ironiste l'a en commun avec l'hypocrisie, avec la vantardise, et pourtant, l'ironiste n'est ni un tartuffe ni un hâbleur ${ }^{32}$ ". En effet, ni tartuffe, ni hâbleur, l'ironiste s'apparente à une sorte de comédien de l'énonciation, à un locuteur momentanément délocuté jouant le rôle - au sens pleinement théâtral du terme - de son alter-ego énonciatif. En vertu de ce dédoublement, il bénéficie - lorsque l'ironie prend la forme du reproche, de l'accusation ou de l'injure - d'une sorte d'immunité qui est aussi une forme d'impunité à l'égard du sujet qui en est la cible : c'est bien moi qui « parle » mais ce n'est pas moi qui le « dis ». Aussi pourra-t-on lui reprocher l'inopportunité de sa mise en scène, mais pas d'avoir tenu des propos qu'il n'a somme toute fait que "lire " dans son "scénario " et dont il ne saurait, par conséquent, être tenu pour responsable. L'ironie naît d'un travestissement de la voix énonciative - " je est un autre " - et relève en cela d'une conception presque theâtrale de l'acte d'énonciation. Car les marques de l'ironie sont, pour une large part, celles de la théâtralité : gestes plus ou moins outrés, courbes tonales emphatiques et contrastées, apartés fictifs, indicateurs mimo-gestuels de toutes sortes, sont autant d'indices verbaux ou paraverbaux par lesquels le locuteur affiche son désengagement à l'égard de l'énonciateur étranger qui, l'espace de quelques secondes, usurpe sa parole ${ }^{33}$.

32. L'ironie, Paris, Librairie Félix Alcan, 1936, p. 44.

33. Pour le problème des indices de l'ironie on consultera avec profit $\mathrm{H}$. Morier, Dictionnaire de poétique et rhétorique, PUF, 1961, p. 578 et s. et tout particulièrement l'étude de Catherine Kerbrat-Orecchioni * Problèmes de l'ironie » in Linguistique et Sémiologie, (op. cit. note 25), p. 10-47. 
2. «...le contraire...»

On pourrait alors, sur la base de cette deuxième partie de la définition de Fontanier, envisager une approche sémantique du phénomène de l'ironie visant à déterminer l'« écart " qui, comme s'accordent à le dire la plupart des spécialistes, oppose les deux contenus énonciatifs - « littéral " et "figuré " - dont le discours est censé être simultanément porteur ${ }^{34}$, c'est-à-dire l'écart entre ce l'« on dit » et ce que l'" on veut faire penser ". Ainsi pourrait-on, par exemple, considérer l'ironie comme le produit d'une sorte de neutralisation rhétorique de l'opposition positif / négatif. Parce que l'énoncé ironique engendre - comme aucun manuel de rhétorique ne manque de le souligner - deux niveaux de lecture contradictoires dont l'un apparaît comme négation inversive de l'autre, l'ironie relève d'une théorie de la modalité qui met directement en cause le double rapport logicoformel du plus et du moins, c'est-à-dire le mécanisme énonciatif de la négation.

En dehors du cadre spécifique de l'ironie, on peut, d'une manière générale, considérer que l'opposition $+/$ - se trouve neutralisée dès lors que le choix dans l'énoncé d'un élément ou de son «contraire " n'entraîne, sur le plan du contenu représentatif, pas de modification sensible dans l'énoncéc ${ }^{35}$. C'est notamment le cas des énoncés dont le prédicat apparaît comme une mise en débat du procès déclaré par le verbe :

34. Cette conception de l'énoncé ironique comme étant investi de deux sens, l'un propre, l'autre figuré, est discutée plus loin.

35. Pour une approche générativiste du phénomène, cf. Ignacio Bosque, Sobre la negación, Madrid, Cátedra, 1980, p. 33 et s. 


$$
\begin{aligned}
& \text { Como se te ocurra decir [ } \left.{ }^{\text {algo }}\right\} \text {, te mato. } \\
& \text { nada }
\end{aligned}
$$

Dudo mucho que así lleguemos a

$\{\underset{\text { ninguna }}{\text { alguna }}\}$ parte.

¿Cuándo me has regalado

Me sorprendería que viniera
[ $\begin{aligned} & \text { algo? } \\ & \text { nada? }\end{aligned}$

1

alguien.

nadie.

On remarquera au passage, en marge de la question qui nous occupe ici, que dans certains contextes la neutralisation du rapport $+/-n$ 'est qu'apparente, la formulation positive ou négative de l'énoncé pouvant répondre à deux manières différentes de concevoir le même procès. Ainsi, comme l'explique Claude Hagège, si l'on compare les formes réduire au maximum et réduire au minimum, on constate, malgré l'invariance de la situation visée (les deux énoncés évoquent également l'idée d'une forte réduction) que les deux compléments ne quantifient pas la même réalité, réduire au maximum s'appliquant à l'opération elle-même et réduire au minimum mettant plus spécifiquement en cause son résultat ${ }^{36}$. De même, dans l'exemple suivant :

36. L'homme de paroles, Fayard, 1986, p. 196. C'est, me semble-t-il, dans une perspective analogue qu'il convient d'analyser les signifiants ya et más lorsque ceux-ci apparaissent dans des contextes négatifs (ya no... / no...más). Faussement synonymiques, ces deux signifiants adverbiaux sont investis d'une valeur démarcative qui, comme dans le cas de leur homologue français plus, instaure une représentation bipartite du procès verbal envisagé, mais qui, à la différence du signifiant français, se trouve doublée d'une visée focale qui oriente la pensée tantôt vers l'accompli, tantôt vers l'inaccompli. En effet, un énoncé tel que je ne fume plus pose négativement que l'action de fumer n'est pas validée dans le présent (je ne fume pas maintenant) en même temps qu'elle présuppose affirmativement qu'elle l'a été par le passé (je fumais auparaoant). Contrairement au français qui, pour signifier l'interruption du procès dispose d'un signifiant unique, l'espagnol s'est doté de deux signifiants différents pour baliser distinctement le terme ad quem de l'accomplissement du procès (c'est-à-dire la fin de l'action : ya no fumo) et le terme a quo de son inaccomplissement (c'est-à-dire le début de l'inaction : no fumo más). Si les deux énoncés sont invariablement rendus en français par je ne fume plus, le premier (avec ya) prend une valeur terminative (je ne fume plus $=\alpha j$ ai cessé de fumer $n$ ) alors que dans le second (avec más) le procès est envisagé sous son aspect inchoatif (je ne fume plus = * j'ai commencé à ne pas fumer $»)$. On évitera toutefois d'assigner - comme on le fait parfois - une valeur " régressive " à ya et une valeur * progressive " à más, les deux 
Cuando se queman intencionalmente $\mathbf{4 5 0 0}$ hectáreas de monte y además se ha detenido al irresponsable, se disipan las dudas que siempre nos hemos planteado.

(Diario de Nazarra, 13-8-1991, p. 19)

on pourrait penser qu'il y a neutralisation puisqu'il est possible de substituer au nom irresponsable son contraire responsable sans que le référent change, mais la responsabilité dont il est question ici n'est pas la même dans les deux cas, le responsable matériel d'une action criminelle étant par là-même et sans contradiction aucune un irresponsable sur le plan moral.

On pourrait donc considérer, suivant ce raisonnement, que de la même manière que l'indéfini nada "équivaut " a son "contraire " algo dans l'énoncé si dices nada me voy, où il se trouve "neutralisé " par son environnement contextuel (à la différence de son emploi dans si no dices nada me voy), le sens littéral de l'énoncé ironique est également "neutralisé" au bénéfice de son sens figuré, auquel il est

signifiants, invariablement prospectifs, étant également orientés en direction du futur. Combiné avec le morphème no, le signifiant ya négative le procès en déclarant le terme de son accomplissement $[=$ cesser de $(-)+$ verbe $(+)]$, alors que más positive le procès en déclarant le début de son inaccomplissement $[=$ commencer à $(+)+$ ne pas verbe $(-)]$. Aussi peut-on percevoir, dans les contextes où les deux signifiants commutent, des variations notables au point de vue de l'énonciation : ya no fumo, par exemple, apparaît comme un énoncé purement constatif ( $j^{\prime} a i$ arrêté de fumer) alors que no fumo más s'apparente à un énoncé promissif (je ne refumerai plus). Sans altérer le rapport d'antériorité et de postériorité qui s'instaure entre les deux moments nécessairement impliqués par ces deux signifiants, l'actualisation de l'un ou de l'autre aura pour effet de mettre en avant soit la cause (ya no tengo dinero : « j’ai dépensé tout mon argent - donc il ne m'en reste plus - ») soit son effet (no tengo más dinero : « il ne me reste plus d'argent - parce que je l'ai entièrement dépensé $-m$ ). C'est parce que ces deux signifiants ne font pas double emploi que le système autorise la co-actualisation des deux formes à l'intérieur du même énoncé pour instituer une négation catégorique (ya no fumo más : négation de l'entier des deux moments impliqués - l'avant et l'après -). On pourrait dire, en termes guillaumiens, que le signifiant ya permet de saisir à son terme le procès (perçu en immanence) dans la limite interne de son accomplissement $\left(l_{1}\right)$ alors quavec más le procès (perçu en transcendance) est saisi dans la subséquence de son extinction (limite externe $1_{2}$ ):

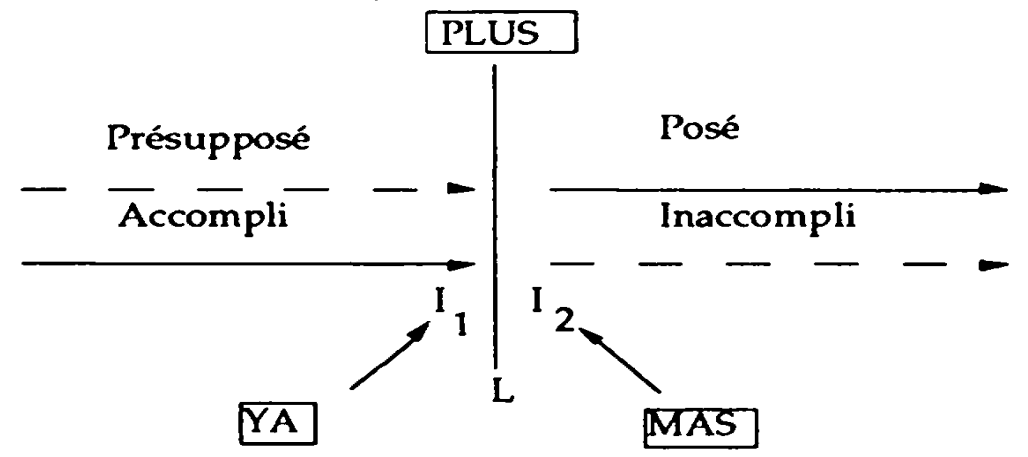


sémantiquement opposé. Ce qui revient à établir entre les deux contenus, littéral et figuré, de l'énoncé ironique un rapport de négation ou d'antonymie phrastique : l'énoncé ironique doit, selon sa définition traditionnelle, être interprété comme étant porteur d'un contenu " contraire " à celui que le discours délivre littéralement.

C'est sans doute le cas lorsque je dis d'un tricheur qu'il est honnête, d'un menteur qu'il est sincère ou de quelqu'un qui est paresseux qu'il est diligent, encore que, insérés dans une situation de parole spécifique, la visée perlocutoire de tous ces adjectifs dépasserait très certainement le sens strict de leur antonyme. Mais on perçoit tout de suite les risques d'une telle restriction. Lorsque dans La linterna mágica de José Tomás de Cuéllar ${ }^{37}$, Natalia, ayant été surprise par Pío Cenizo alors qu'elle s'amusait en compagnie d'Isaura et de Rebeca à un jeu quelque peu puéril, prétexte pour se justifier devant celui-ci que le canari s'est échappé de sa cage, on ne saurait interpréter l'exclamation i $Q u e ́$ lástima! par laquelle rétorque ironiquement Pío Cenizo comme une manifestation quelconque de joie ou de soulagement ( $i$ Qué alegría !), mais simplement comme l'expression de son incrédulité. Comme le fait remarquer Philippe Hamon, "le sens visé par une phrase ironique comme "Je suis vraiment tout a fait ravi d'apprendre cela" n'est pas forcément "Je suis tout à fait peiné d'apprendre cela", mais sans doute "Cela m'est tout à fait indifférent d'apprendre cela" „38.

L'opération qui sous-tend l'énonciation ironique ne se laisse donc pas ramener à une simple inversion sémantique qui permettrait d'accéder automatiquement au sens figuré de l'énoncé en négativant son sens littéral. Le rapport qui oppose les deux valeurs véhiculées par l'énoncé ironique n'est pas, comme on le dit souvent encore, un rapport antiphrastique ou $d^{\prime}$ '" antonymie sémantique " - a supposer, comme le fait remarquer à juste titre Catherine Kerbrat-Orecchioni, que l'on puisse parler de phrases antonymiques ${ }^{39}$-, mais bien plutôt un rapport d'«antonymie focale ": ce qui s'oppose dans l'acte d'énonciation ironique, ce ne sont pas deux contenus sémantiques, positif et littéral l'un, négatif et figuré l'autre, mais deux points de vue divergents : celui d'un locuteur et celui d'un énonciateur antagoniste. En identifiant

37. Exemple donné par Helena Beristáin (Diccionario de retórica y poética, México, Editorial Porrúa, 1988, p. 272) pour illustrer le phénomène de l'anticatastase.

38. * L'ironie » in Le grand atlas des littératures, Enciclopædia Universalis, 1990, p. 56.

39. Pour les notions de * contraire » et d'* antonyme » cf. notre étude * La négation antiphonique en espagnol. La formule de renforcement $\mathrm{Ni}$ insulas ni insulos. Etude synchronique et diachronique $m$ in Bulletin Hispanique, 95, 1992, num. 2. 
ironie et antiphrase ${ }^{40}$, la rhétorique traditionnelle fait de l'antonymie le principe fondateur de l'énonciation ironique; elle postule ainsi pour caractériser le trope l'existence de deux dicta opposés ( littéral o ou «propre » et « dérivé » ou « figuré ») à la fois énoncés et assumés par un seul et unique locuteur, alors que ce sont deux énonciateurs distincts qui, devant le même dictum, s'affrontent par des prises de position contrastées. En d'autres termes, l'ironie ne consiste pas à dire le contraire de ce que l'on veut faire entendre, mais a faire adopter a un énonciateur fictif un point de vue différent de celui quadopte le producteur matériel de l'énoncé à l'égard du même et unique dit.

C'est pourquoi il n'y a pas lieu de parler, comme on le fait habituellement, de deux sens différents, l'un "littéral ", l'autre «figuré», qui, comme dans le cas par exemple de la métaphore, coexisteraient dans le même énoncé ou qui se substitueraient l'un a l'autre. Le fait qu'il présente une " double lecture " ne veut aucunement dire que l'énoncé ironique soit porteur de deux sens, c'esta-dire de deux contenus sémantiques différents : ce qui se trouve dédoublé dans l'énonciation ironique c'est le point de vue énonciatif et non le sens de l'énoncé. Ainsi, pour reprendre un exemple devenu banal, en disant par une journée particulièrement pluvieuse $Q$ u el temps splendide!, le locuteur ne dit ni ne veut faire entendre autre chose que ce que déclare très exactement l'assertion qu'il vient de proférer. Pourquoi proférerait-il une telle phrase s'il ne souhaitait pas que son interlocuteur entende très exactement ce que dit littéralement l'énoncé? Certes, le caractère scandaleux de l'assertion par rapport à la situation extra-linguistique autorise à interpréter de différentes manières, non pas le contenu de l'énoncé, mais l'intentionnalité de l'énonciateur, qui peut vouloir exprimer ainsi sa contrariété devant la situation météorologique ou, comme l'a suggéré Morier, en conjurer imaginairement les effets en feignant d'ignorer cette situation. Mais on peut tout aussi bien imaginer que le locuteur a voulu ridiculiser un passant se promenant sous la pluie en tenue estivale ou encore reprocher à son interlocuteur son esprit de contradiction s'il le sait enclin à nier l'évidence. On ne saurait pourtant considérer toutes ces "lectures " comme des sens dérivés ou figurés, ni même comme des sens; l'énoncé ironique a beau susciter des interprétations multiples, son sens, invariablement " propre " et « littéral"1 ", reste inchangé : ce

40. C'est ce que fait le groupe $\mu$ (Rhétorique générale, Paris, Editions du Seuil, 1982), pour qui * l'ironie montre mieux [que l'euphémisme] quelle distance on peut prendre à l'égard des faits, car presque toujours elle les nie. L'antiphrase s'en distingue à peine » (p. 139).

41. * L'esprit supplée, corrige, interpole, perçoit enfin cette ponctuation secrète qui est au-delà de la phrase visible [...] Pourtant ce sont encore des mots qui servent à nous faire 
n'est pas le sens de l'énoncé qui offre plusieurs lectures, mais l'intention de celui qui le profère ${ }^{42}$. Partant du principe que l'énoncé ironique était investi de deux sens ${ }^{43}$, on a voulu voir une figure de substitution là où il n'y avait qu'une figure d'intention, ce que l'on a traditionnellement appelé sens figuré n'étant autre chose que l'intentionnalité du locuteur ironique. En effet, pour qu'il puisse y avoir substitution, il faut que les éléments permutés soient sinon de même nature du moins comparables : on pourra, si on le souhaite, " remplacer" un mot par un autre ou un sens par un autre, mais pas le sens d'un énoncé par l'intention de celui qui l'énonce. En traitant l'énoncé ironique comme un énoncé polysémique on a tout simplement assimilé le contenu sémantique de la phrase à l'effet virtuellement visé ou effectivement suscité par son énonciateur dans un contexte ou dans une situation donnés, mettant ainsi sur le compte de la sémantique ce qui est du seul ressort de la pragmatique.

oublier les mots; pour volatiliser la matière, il nous faut d'abord accepter la matière, et l'artiste le sait bien, qui s'installe dans le monde des corps figurés. En cela consiste, selon nous, l'acrobatie du "style" : à tout moment nous devinons, derrière la lettre, la secrète respiration de l'esprit, et à tout moment le poids de la lettre risque de nous retenir au sol. Pour lire ce qui n'est pas écrit, pour entendre ce qui n'est pas proféré, il faut que le silence se peuple " (Vladimir Jankélévitch, ibid., p. 36). La distinction entre * lettre » et * esprit de la lettre » à laquelle fait appel Jankélévitch, présente au moins l'avantage de ne pas assimiler les différentes valeurs que l'on peut attacher à l'énoncé ironique à un contenu sémantique figuré dérivé de et substituable à un contenu sémantique littéral. La remarque suivante de Graciela Reyes à propos du sens littéral de la phrase ironique nous semble à cet égard tout à fait pertinente : * No es acertado afirmar, según es corriente, que el locutor irónico quiere decir exclusivamente lo que no dice : también quiere decir lo que dice, puesto que usa de lo que dice para decir por añadidura lo que no dice " (Polifonía textual. La citación en el relato, Madrid, Gredos, 1984, p. 158).

42. On pourra objecter ici qu'à la base de toutes ces interprétations se trouve un seul énoncé virtuel, par exemple quel sale temps!, qui correspondrait bien au « sens figuré » de l'énoncé ironique quel temps splendide! On remarquera cependant que quel sale temps! (qui n'est pas un sens mais un énoncé) n'est en fait qu'une paraphrase explicative sujette à toutes sortes de variations (quel mauoais temps, il pleut, je n'aime pas la pluie, etc.). Il n'est d'ailleurs pas nécessaire d'inverser le sens de l'énoncé ironique pour comprendre ce que * veut dire * le locuteur : dans le passage cité de La Regenta, l'énoncé una madre es un perro ne se laisse pas interpréter comme yo creo que una madre no es un perro, mais bien plutôt comme tú te has creído que una madre es un perro.

43. Catherine Kerbrat-Orecchioni, qui a pourtant défini avec pertinence l'ironie comme * une espèce de trope sémantico-pragmatique " et qui consacre une partie de son analyse à la notion d'* intentionnalité ", insiste à opposer deux niveaux de sens : le * sens littéral (premier, patent, inscrit dans la langue) $=$ connoté " et le * sens dérivé (second, latent, plus ou moins inédit) = dénoté " ( "Lironie comme trope " in Poétique, num. 4, 1980, p. 109). 


\section{3. *...de ce que l'on pense, ou de ce que l'on veut faire penser. *}

C'est précisément cette notion d'intentionnalité qu'introduit la dernière partie de la définition proposé par Fontanier. Mais en faisant appel pour caractériser le trope à des paramètres aussi différents que la pensée du locuteur et son intention, l'auteur mêle dans sa définition deux notions différentes, a savoir : l'hypocrisie, qui effectivement consiste à dire le contraire de se que l'on pense, et l'ironie, qui consiste à dire sinon le contraire de ce que l'on veut faire entendre, du moins autre chose que ce que l'on souhaite transmettre. En d'autres termes, la définition de Fontanier n'établit aucune distinction entre l'ironie et le mensonge, notions apparemment proches, mais radicalement opposées tant par leur nature que par leur objet. Ainsi, contrairement au menteur, qui feint de dire la vérite, l'ironiste, lui, feint de faire un mensonge : le premier ment sans le montrer, le second montre qu'il ment; le menteur cache, l'ironiste exhibe, l'un dissimule, l'autre ne fait que simuler. D'où le nom de simulatio par lequel, avec celui d'illusio, la rhétorique classique désigne le trope. L'ironie est certes un mensonge, mais - et c'est en quoi elle s'en distingue - un mensonge qui s'affiche comme tel. C'est une telle confusion qui, par exemple, amène Kurt Spang à inclure dans son manuel de rhétorique ${ }^{44}$, pour illustrer le trope, le passage suivant de La Célestine :

AREUSA. ¿Quién anda ay ? ¿Quién sube á tal hora en mi cámara ?

CELESTINA. Quien no te quiere mal, cierto; quien nunca da passo que no piense en tu prouecho; quien tiene mas memoria de tí, que de sí mesma : vna enamorada tuya, avnque vieja. (VII)

Mais on le voit bien : si le discours de Célestine avait, comme le prétend K. Spang, une visée effectivement ironique, cette réplique constituerait une véritable auto-accusation de la part du personnage. Si Célestine dit "le contraire » de ce qu'« elle pense ", ce n'est pas pour dévoiler ses pensées, mais bien au contraire pour les cacher : loin d'ironiser, elle ne fait ici que mentir hypocritement à Areúsa.

Si ce que pense le locuteur ironique ne devait pas transparaître dans son discours, si l'énonciation polyphonique ne devait y laisser aucune trace, alors non seulement l'ironie serait indétectable, mais son étude en tant que phénomène rhétorique, énonciatif ou pragmatique, ne se justifierait plus. Car il faut, pour que l'ironie soit perçue comme telle, que le locuteur affiche son intention d'ironiser sans renoncer pour autant à une certaine ambiguïté, l'équivoque étant souvent l'objet même de l'énonciation ironique : « ...l'ironie - explique Catherine 
Kerbrat-Orecchioni - ne se justifie que dans la mesure où elle reste au moins partiellement ambiguë : quel intérêt y aurait-il à parler ironiquement si c'est pour immédiatement rectifier le tir en spécifiant ce que l'on veut vraiment dire ? L'ironie ne peut légitimement exister qu'en l'absence d'indices trop insistants [...] du leurre qu'elle constitue $\gg 45$.

Se pose ainsi le problème des marques formelles ${ }^{46}$ de l'ironie dans le discours oral et, tout particulièrement, dans le discours écrit, qui ne peut, sauf de maniere implicite ou par voie de didascalie, rendre compte de phénomènes discriminateurs comme l'intonation, la modulation de la voix, les gestes ou la prononciation. On connaît les différentes tentatives qui, secondées par écrivains et imprimeurs, ont vu le jour pour instaurer un signe de ponctuation supplémentaire qui permettrait de rendre "visibles " les indices oraux de l'énonciation ironique, tel le fameux "point d'ironie ", plusieurs fois inventé au cours de l'histoire et adopté par le Mulhousien Alcanter de Brahm (1868-1942) qui le matérialisa dans son Ostensoir des Ironies (1899-1900) par le signe / $\ / 47$. Mais comme le fait remarquer Henri Morier, "ce serait aller plus loin que ne le fait la langue parlée, où les méprises sont également possibles ${ }^{48} "$. Remarquons toutefois que des contraintes tout aussi lourdes se font sentir lorsqu'on passe de la langue écrite a la langue orale: en l'absence de signes d'assise "audibles", le recours à des locutions comme entre comillas ou entre guillemets - souvent accompagnées d'un geste -, qui font office dans la langue parlée de véritables "guillemets sonores", témoigne d'une singulière incursion du discours écrit dans le code oral.

L'une des marques - audibles et quantifiables - de l'énonciation polyphonique en régime oral est l'intonation, phénomène complexe

45. Ibidem, p. 109. * Lironiste [...] se cache, mais pas trop, pour qu'on ait envie de le trouver... » (V. Jankélévitch, op. cit., p. 53).

46. Cf. supra note 33.

47. L'Ostensoir des Ironies. Essai de métacritique, Paris, Bibliothèque d'Art de la Critique, 1899 (Première partie : « L'homme, la femme \& la famille "), 1900 (Deuxième partie : « La société "; Troisième partie : * Les étapes de la pensée et le sens de la vie »). En voici quelques extraits : « Patrie et Religion apparaissent comme les nœuds coulants strangulateurs de la liberté individuelle; et le Capital vient à point pour achever leur ouvre néfaste $\}$ " (I, p. 4); « Les nihilistes sont vraiment des gens très raisonnables ? " (II, p. 86); * Il semble que la philosophie et la dialectique soient devenues jeux d'oisifs et bons tout au plus à rendre les rhétoriciens impatients de se jeter à leur tour en la mêlée. La Science triomphe ? (III, p. 97). Cf. également Jacques Drillon, Traité de la ponctuation française, Gallimard, 1991, p. 11-12.

48. Ibidem, p. 217. 
que nos signes de ponctuation, si vagues et si peu diversifiés, s'attachent à représenter, même si le plus souvent ils ne font, compte tenu de leur surcharge fonctionnelle, qu'en donner l'illusion. Or l'étude du phénomène intonationnel - dont certaines réalisations peuvent parfois jouer dans l'énoncé le rôle de véritables morphèmes prosodiques - ne peut être efficace que si elle s'appuie sur l'analyse concrète d'énoncés réels produits dans des situations de parole réelles. Habitué cependant à raisonner à partir d'énoncés artificiellement isolés et à s'en représenter abstraitement les courbes mélodiques en fonction moins de l'observation directe des faits que de sa compétence et de sa connaissance intériorisée de la langue, il arrive parfois a l'analyste d'imputer aux unités suprasegmentales ce qui, de fait, relève de la seule situation d'énonciation ou, parfois, du contexte discursif lui-même. Ainsi dans son essai sur la Polifonia textual, au chapitre qu'elle consacre au phénomène de l'ironie, Graciela Reyes écrit :

Una a miga me cuenta una serie de pequeñas desgracias cotidianas. Dice que después de muchos accidentes llega por fin, sin aliento, a la oficina en que debía hacer un trámite urgente : Llegué a las dos menos diez y ya habian cerrado. Me ha dado un gusto. La última frase se dice con entonación ligeramente ascendente, distinta de la entonación del resto, y una pausa para que yo, interlocutora, "añada " el significado que hay que añadir, la implicatura correspondiente. * Me dio una rabia " hubiera transmitido en este caso lo mismo, poco más o menos, pero la ironía tiene la ventaja de que obliga al interlocutor a participar ${ }^{49}$.

Que l'on remplace Llegué a las dos menos diez y ya habían cerrado par Me ha tocado la quiniela et l'on se persuadera aisément qu'aucune trace matérielle de l'ironie ne transparaît au niveau des suprasegments dans l'émission de la phrase Me ha dado un gusto : que l'on dise - ironiquement ou non, d'ailleurs - Me ha dado un gusto ou Me ha dado una rabia, la courbe intonative reste rigoureusement la même dans les deux cas. Loin de constituer, comme le prétend Graciela Reyes, l'indice d'un dédoublement polyphonique, le tonème ascendant a pour seule fonction d'indiquer ici que l'énoncé est syntaxiquement tronqué et qu'il réclame pour être complet soit un complément adjectival soit un complément de manière ou de conséquence (me ha dado un gusto / una rabia...enorme / que ni te cuento / que no veas / que para qué, etc.): seule la prise en compte du contexte énonciatif - ici dévoilé d'emblée par le préambule explicatif de l'auteur - permet de reconnaître l'intentionnalité ironique qui sous-tend l'énonciation, l'intonation étant à cet égard tout à fait indifférente.

49. Ibidem, p. 160. 
L'intonation, dont on ne saurait bien évidemment nier l'incontestable pouvoir à discriminer des valeurs énonciatives différentes et à lever l'ambiguïté de certains énoncés, ne joue pas toujours, comme on le voit, le rôle discriminateur qu'on lui accorde. Ainsi une expression aussi banale et en apparence inéquivoque que la locution espagnole si hombre s'avère-t-elle apte à signifier, sans la moindre modification tonale, aussi bien l'approbation que le refus le plus catégorique. A une question comme i Te gustaría volver a vivir esa experiencia? le locuteur pourra répondre affirmativement ou négativement, selon qu'il jugera l'expérience en question bonne ou mauvaise, par la locution sí hombre, la valeur de la réponse étant fonction ici de facteurs purement pragmatiques; et si, souvent, l'intonation qui accompagne cette réplique ne laisse pas subsiter le moindre doute quant aux intentions du locuteur, elle est parfois ambiguë au point que, isolée de son contexte et entendue, par exemple, sur un enregistrement, il n'est pas toujours aisé de déterminer si la réplique est ironique ou non. En revanche, l'énonciation ironique s'accompagne parfois de faits articulatoires notables, auxquels les manuels de phonétique ne prêtent pas d'attention particulière. Il est curieux de constater, par exemple, que le locuteur hispanophone à qui il est parfois si difficile de reproduire le timbre de la voyelle française $u$ (on connaît la boutade des lycéens espagnols s'initiant à l'étude du grec classique qui, prononçant ipsilon au lieu de upsilon, plaisantent sur la "liosa " conjugaison du verbe $\lambda v \omega)$, peut dans certaines situations émettre un son labialisé proche du $u$ français, timbre vocalique dont les phonéticiens s'accordent pour dire qu'il n'existe pas en espagnol. Car aussi étrange que cela puisse paraître, la réalisation arrondie [y] non pas de la voyelle postérieure / $\mathrm{u} /$ mais de la voyelle antérieure /i/ n'est pas vraiment étrangère au système articulatoire de l'espagnol, même si le phénomène ne concerne a ma connaissance, tout au moins dans sa manifestation la plus courante, qu'un seul et unique mot : le signifiant adverbial sí qui, en particulier dans la réponse ironique - donc employé pour dire le refus -, est souvent articulé [sy]. Cette labialisation constitue essentiellement un procédé articulatoire d'insistance. Ainsi, il y a dans certains contextes, entre la réalisation [si] et sa variante labialisée [sy], un écart énonciatif aussi fort que celui qui peut opposer le mot oui, simple réponse affirmative donnée par le locuteur à une demande d'information formulée par un autre locuteur, a la locution bien sûr, réplique donnée à une question qui, implicitement jugée nonpertinente par le sujet interrogé, est en quelque sorte invalidée rétroactivement, car elle laisse sous-entendre que toute autre réponse était, dans l'esprit du locuteur, inenvisageable. Il reste toutefois que si la réalisation labialisée de ce mot fonctionne souvent comme une marque de l'ironie, cette valeur ne lui est nullement exclusive, la forme [sy] 
pouvant apparaître dans des contextes variables pour indiquer, en combinaison avec des courbes tonales spécifiques, l'insistance, la surprise, l'affirmation atténuée ou l'indifférence.

Nombreux et divers, les marqueurs de l'ironie, souvent appelés à fonctionner concurremment comme des complexes indiciels à l'intérieur d'un même énoncé, constituent un dispositif de "mise en alerte " efficace, mais nullement infaillible. Enonciation a risque, l'ironie qui, comme l'a souligné Alain Berrendonner ${ }^{50}$, permet de contourner la norme sociale et de mettre le locuteur à l'abri de toute sanction, peut se retourner dangereusement contre lui - effet pervers que Graciela Reyes appelle ironía abortada ${ }^{51}$ - si elle n'est pas perçue comme telle par l'auditeur : que l'on pense, par exemple, a un compliment fait par antiphrase ou à l'astéisme de la rhétorique classique. L'ironie, comme l'intertextualité, demande pour être reconnue que l'émetteur et le récepteur participent du même code : de la même manière que la présence dans le discours littéraire d'indices intertextuels ne peut être décelée que par le lecteur possédant une certaine " compétence littéraire ", la détection de l'ironie passe par une certaine « compétence idéologique ${ }^{52}$ " que locuteur et destinataire

50. * Il m'importe peu que l'ironie soit morale ou non. L'essentiel est [...] qu'il s'agit d'une manouvre à fonction fondamentalement défensioe. Et qui plus est, défensive contre les normes. Elle apparaît [...] comme une ruse permettant de déjouer l'assujettissement des énonciateurs aux règles de la rationalité et de la bienséance publiques. Elle représente donc un moyen - peut-être le seul - qu'ait l'individu parlant de s'affranchir d'une contrainte normative, sans avoir à subir les sanctions qu'entraînerait une franche infraction. Contre le "fascisme" que Barthes, par une hyperbole elle-même suspecte d'ironie, reprochait naguère aux normes du langage, l'ironie fait figure de réplique "antifasciste". Car elle peut apparaître, dans l'ordre de la parole, comme le dernier refuge de la liberté individuelle $m$, Alain Berrendonner, Eléments de pragmatique linguistique, Paris, Les Editions de Minuit, 1981, p. 239.

51. Op. cit., p. 178. V. Jankélévitch a également insisté sur les risques encourus par l'énonciateur ironique : « On ne saurait s'étonner que l'ironie offre certains dangers, tant pour l'ironiste lui-même que pour ses victimes. La manceuvre est risquée et, comme tout jeu dialectique, elle ne réussit que de justesse : un millimètre en deçà - et l'ironiste est la risée des hypocrites ; un millimètre au-delà - et il se trompe lui-même avec ses propres victimes... " (op. cit., p. 98).

52. A propos de cette « compétence idéologique " qui doit permettre à l'auditeur d'avoir accès au * système de valeurs implicites » de l'émetteur, Philippe Hamon explique : « L'étranger, on le sait, même celui qui possède parfaitement notre langue [...], cest celui qui ne rit pas des choses dont il devrait rire et qui rit des choses dont il ne devrait pas rire [...] Le I Iuron de Voltaire, le Persan de Montesquieu, le Provincial de Pascal, Bouvard et Pécuchet chez Flaubert, les héros de La Plaisanterie de Milan Kundera, le Petit Prince de Saint-Exupéry ou le Gulliver de Swift sont tous, d'un certain point de vue, des étrangers confrontés à des systèmes de valeurs ou à des coutumes auxquels ils n'ont que partiellement accès " (ibid., p. 56). C'est dans cette même lignée que s'inscrit l'héroïne de La tesis de Nancy et de Epilogo a Nancy de Ramón J. Sender dont l'écriture exploite massivement le procédé de l'ironie. Sur les problèmes spécifiques 
doivent nécessairement partager. L'ironie entretient par ailleurs avec l'intertextualité des rapports étroits puisque les deux phénomènes relevent d'une pratique citationnelle analogue. Ainsi que D. Sperber et D. Wilson l'ont mis en évidence, l'acte ironique est un acte de mention, puisque le locuteur cite ou feint de citer un énoncé qui émerge dans son discours comme un "écho plus ou moins lointain, de pensées ou de propos, réels ou imaginaires, attribués ou non à des individus précis ${ }^{53}$ ». Mais a la différence de la citation intertextuelle, qui opere effectivement la réécriture d'un discours antérieur, l'ironie ne fait que la simuler : le discours ironique serait, pour reprendre la terminologie de Genette, une sorte d'hypertexte dont l'hypotexte n'existe que dans l'esprit du locuteur qui, au gré de ses besoins argumentatifs, improvise ses propres sources et leur confère le statut de citations. Ironiser, c'est, en somme, reprendre un discours qui paradoxalement ne préexiste pas à sa reprise ; ce qui n'interdit pas d'enchâsser une structure citationnelle dans une mention ironique, comme l'illustre le dialogue suivant :

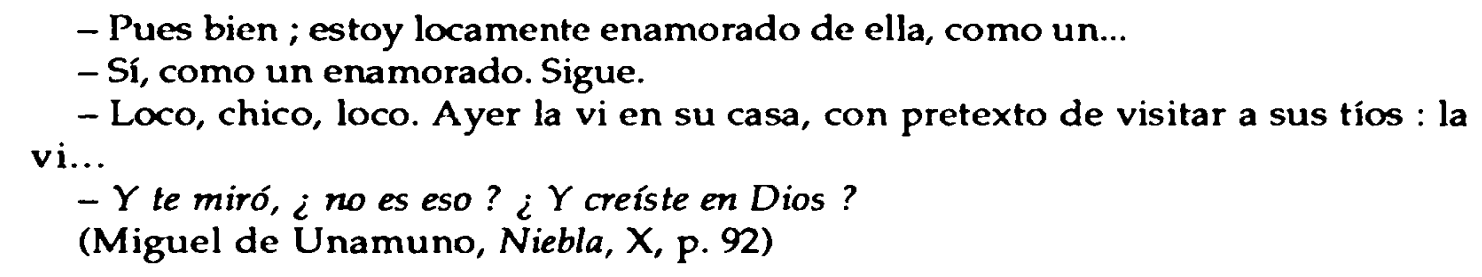

Discours polyphonique à trois voix, la dernière réplique est celle d'un locuteur " mentionnant " un énonciateur " détournant " parodiquement les vers bien connus de Bécquer.

L'ironie apparaît ainsi comme le lieu d'une subversion - codifiée et exhibée - des lois de l'assertion. Que l'on considère l'ironie comme un trope (Catherine Kerbrat-Orecchioni) ou comme une mention (D.Sperber, D. Wilson), comme une " contradiction de valeur argumentative" (Alain Berrendoner) ou comme une simple construction antiphrastique (Pierre Fontanier), c'est, en dernière

de la contextualisation littéraire de lironie, considérée comme phénomène portant non pas sur un mot ou sur un énoncé isolé mais sur l'ensemble de l'œuvre littéraire, on se reportera utilement à l'étude de Linda Hutcheon * Ironie, satire, parodie. Une approche pragmatique de l'ironie " in Poétique, num. 46, p. 140-155.

53. « Nous soutenons que toutes les ironies typiques, mais aussi bon nombre d'ironies atypiques du point de vue classique, peuvent être décrites comme des mentions (généralement implicites) de propositions; ces mentions sont interprétées comme l'écho d'un énoncé ou d'une pensée dont le locuteur entend souligner le manque de pertinence " (Dan Sperber et Deirdre Wilson, * Les ironies comme mentions " in Poétique, num. 36, 1978, p. 409). 
instance, le dédoublement polyphonique du sujet parlant, scindé en deux voix distinctes qui se font cependant entendre simultanément, qui spécifie l'énonciation ironique et explique son fonctionnement paradoxal. Acte de parole " apocryphe ", l'énonciation ironique est le theâtre d'une imposture énonciative qui, montrée et mise à nu par l'énonciateur lui-même, permet au locuteur de se soustraire imaginairement, en travestissant sa voix, à son propre je locutif : en cela, l'ironie apparaît comme une forme d'énonciation déguisée qui, pour reprendre la pensée de Bahktine à qui l'on doit le concept de polyphonie, mériterait bien le qualificatif de « carnavalesque ".

Les trois repères fondateurs de l'acte d'énonciation (la personne locutive - je -, le lieu - ici -, le temps - maintenant -) déterminent donc une position de locution a laquelle, comme on l'a vu, l'énonciateur n'est aucunement tenu d'adhérer. En se désolidarisant de la position du locuteur, l'énonciateur, qui s'en distingue a peine lorsqu'il fait sien ce triple repère (c'est-a-dire lorsque la position de locution et la position d'énonciation coöncident), clive l'instance de locution détournant ainsi l'objet d'énonciation vers l'acte d'énonciation lui-même. Au contenu représentatif proprement dit s'ajoute donc dans l'énoncé polyphonique le point de vue énonciatif adopté par le sujet parlant à l'endroit de son propre dit. L'effet ironique qui résulte de la prise de distance de l'énonciateur à l'égard de sa propre assertion ou l'effet de " parallaxe " produit par le déplacement spatiotemporel de la voix énonciatrice, ne sont en dernière analyse que des manifestations particulières d'un même et unique processus énonciatif déclenché par le refus de l'énonciateur à partager avec le locuteur le point de vue personnel ou spatio-temporel du sujet parlant. Soit, en figure :

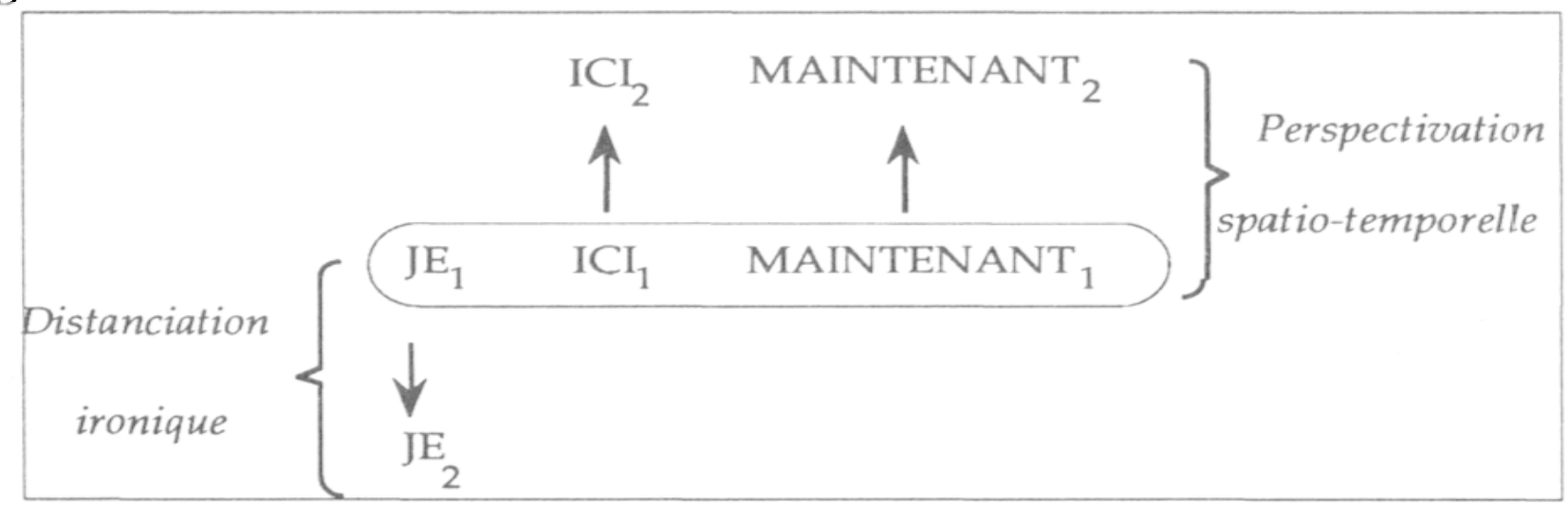


L'interprétation de l'énoncé polyphonique passe ainsi par la reconnaisance d'un locuteur dédoublé se donnant, en même temps qu'une représentation du monde, celle du système qui lui sert à le représenter. Les ruptures que semble générer, dans l'ordre linguistique, pragmatique ou idéologique du discours, le clivage de l'instance de parole ne sauraient masquer la cohérence interne de l'énoncé polyphonique, cohérence qui, j'ai essayé de le montrer, n'est à rechercher ni dans la conformité du "dit " au réel extralinguistique (l'énoncé ironique ne résiste pas le plus souvent à une telle confrontation avec l'univers référentiel) ni dans celle du " dire " aux règles grammaticales (l'énoncé perspectivé les enfreint largement), mais dans l'intégration au sein de l'énoncé des deux positions de locution et d'énonciation mises en jeu par l'instance locutive. Au-delà des ruptures et des distorsions dont elle semble être a l'origine, l'aptitude de l'énonciateur à se distancer de sa propre production est un facteur puissant de cohésion énonciative. 


\section{REFERENCES BIBLIOGRAPHIQUES}

Alas Clarín (Leopoldo) : La Regenta, Madrid, Alianza Editorial, 1979.

Bello (Andrés) : Gramática de la lengua castellana destinada al uso de los americanos, Paris, A. Roger y F. Chernoviz Editores, 1898.

Bouzet (Jean) : Grammaire espagnole, Paris, Belin, 1974.

Berceo (Gonzalo de) : Signos que aparecerán antes del juicio final (ed. de Arturo Ramoneda), Madrid, Editorial Castalia, 1980.

Carpentier (Alejo) : El siglo de las luces (ed. de Ambrosio Fornet), Madrid, Ediciones Cátedra, 1985.

Carroll (Lewis) : CEuvres (trad. fr. de Henri Parisot), Paris, Robert Laffont, 1989.

Cortázar (Julio) : Los relatos 2. Juegos, Madrid, Alianza Editorial, 1976.

- Los relatos 3. Pasajes, Madrid, Alianza Editorial, 1990.

Coste (J.) \& Redondo (A) : Syntaxe de l'espagnol moderne, Paris, Sedes, 1965.

Delibes (Miguel) : Los santos inocentes, Barcelona, Editorial Planeta, 1992.

Fernández Ramírez (Salvador) : Gramática española. Los sonidos, el nombre y el pronombre, Madrid, Revista de Occidente, 1951.

Góngora (Luis de) : Sonetos completos (ed. de Biruté Ciplijauskaité), Madrid, Editorial Castalia, 1975.

Grevisse (Maurice) : Le bon usage, Paris, Editions Duculot, 1980.

Mauriac (François) : La fin de la nuit, Editions Bernard Grasset, 1935.

Pereda (José María de) : Obras Completas (ed. de José María de Cossío), Madrid, Aguilar, 1940.

Pérez de Ayala (Ramón) : Belarmino y Apolonio (ed. de Andrés Amorós), Madrid, Ediciones Cátedra, 1981.

Sábato (Ernesto) : Sobre héroes y tumbas, Barcelona, Editorial Seix Barral, 1984.

Stendhal : Lucien Leuwen (éd. de Henri Martineau), Paris, Gallimard, 1986.

- Luciano Leuwen (trad. esp. de Consuelo Berges), Madrid, Alianza Editorial, 1981.

Unamuno (Miguel de) : Niebla (ed. de Harriet S. Stevens \& Ricardo Gullón), Madrid, Taurus Ediciones, 1979.

Valle-Inclán (Ramón del) : Obras completas, I, Madrid, Editorial Plenitud, 1952.

Vallejo (César) : Trilce (ed. de Julio Ortega), Madrid, Ediciones Cátedra, 1991. 